\title{
A FINITE ELEMENT METHOD FOR STIFFENED PLATES
}

\author{
Ricardo Durán ${ }^{1}$, Rodolfo Rodríguez ${ }^{2}$ And Frank SAnhueza ${ }^{3}$
}

\begin{abstract}
The aim of this paper is to analyze a low order finite element method for a stiffened plate. The plate is modeled by Reissner-Mindlin equations and the stiffener by Timoshenko beams equations. The resulting problem is shown to be well posed. In the case of concentric stiffeners it decouples into two problems, one for the in-plane plate deformation and the other for the bending of the plate. The analysis and discretization of the first one is straightforward. The second one is shown to have a solution bounded above and below independently of the thickness of the plate. A discretization based on DL3 finite elements combined with ad-hoc elements for the stiffener is proposed. Optimal order error estimates are proved for displacements, rotations and shear stresses for the plate and the stiffener. Numerical tests are reported in order to assess the performance of the method. These numerical computations demonstrate that the error estimates are independent of the thickness, providing a numerical evidence that the method is locking-free.
\end{abstract}

Mathematics Subject Classification. 65N30, 74K10, 74K20.

Received October 29, 2010.

Published online October 12, 2011

\section{INTRODUCTION}

A stiffened plate is a plate reinforced with ribs to increase it capacity to resist loads. Such plates are used typically in buildings, hulls of ships, aircraft and many other applications in the structural engineering.

Different models of stiffened plates have been used. See for instance [12] for a discussion of several simple engineering models and further references. Another approach has been proposed in [15]. It consists of coupling Kirchhoff-Love equations for the plate with Euler-Bernoulli equations for the stiffener. The constraint between both structures is imposed by means of Lagrange multipliers. A particular finite element method is proposed. Numerical experiments demonstrate the effectiveness of this approach although no analysis is given. The same problem has been analyzed in [6] based in a model proposed in [13]. In this case $\mathcal{C}^{1}$-elements are proposed for the plate combined with $\mathcal{P}_{2}-\mathcal{P}_{3}$ elements for the beam. A domain decomposition technique is proposed for the solution of the resulting algebraic problem.

An alternative modeling approach consists of using Reissner-Mindlin equations for the plate and Timoshenko beam equations for the stiffener. This model has been considered in [14], where an elementary finite element method is proposed. However this method is shown to suffer from locking.

\footnotetext{
Keywords and phrases. Stiffened plates, Reissner-Mindlin model, Timoshenko beam, finite elements, error estimates, locking.

1 Departamento de Matemática, Facultad de Ciencias Exactas y Naturales, Universidad de Buenos Aires, 1428 Buenos Aires, Argentina. rduran@dm.uba.ar

2 CI2MA, Departamento de Ingeniería Matemática, Universidad de Concepción, Casilla 160-C, Chile. rodolfo@ing-mat.udec.cl

3 Escuela de Obras Civiles, Universidad Andres Bello, Autopista Concepción, Talcahuano 7100, Concepción, Chile.

fsanhueza@unab.cl
} 
Locking consists in that very unsatisfactory results are obtained when the thickness is small with respect to the other dimension of the structure. From the point of view of the numerical analysis, locking reveals itself in that the a priori estimates depends on the thickness of the structure in a such way that they degenerate when this parameter become small.

Several finite elements have been proposed to avoid locking in Reissner-Mindlin equations (see [9] for a review on this subject). A typical locking-free finite element method, MITC9, which was introduced in [4], is proposed in [12] to discretize the stiffened plate equations. These elements are combined with standard quadratic element for the stiffener. Once more, only numerical evidence of the performance of the method is reported.

In the present paper we use the same model, based on Reissner-Mindlin equations for the plate and Timoshenko equations for the stiffener. We introduce the constraint between both structures by means of Lagrange multipliers. We prove existence and uniqueness of solution to the resulting problem.

A particular case of stiffened plates is when the mid-line of the stiffener lies on the mid-surface of the plate. In this case the plate is known as concentrically stiffened and the problem decomposes into two simpler problems as it happens for unstiffened plates: the in-plane and the bending plate problems. The analysis of the former is quite simple since it leads to equations independent of the plate thickness. This is not the case with the bending problem for the stiffened plate.

In this case we consider a family of problems parametrized by the plate thickness $t$. We show that the mechanical coefficients of the stiffener must be taken proportional to $1 / t$ to obtain a significant limit problem as $t$ goes to zero. We show that the solution of the stiffened plate bending problem is bounded above and below far from zero, independently of $t$. We also prove additional regularity of the solution, although not independently of $t$.

For the discretization of the in-plane stiffened plate we use standard linear elements, and the analysis is straightforward. For the stiffened plate bending problem we use a low order plate finite element, DL3 [7], combined with $\mathcal{P}_{2}-\mathcal{P}_{1}$ elements for the stiffener. The choice of these hybrid elements for the rod allows us to impose the constraint in an simpler way.

We prove optimal order error estimates depending on higher order norms of the solution. These higher order norms are the same that appear in the case of uncoupled plates and rods. In such a case they are bounded independently of $t$. For the stiffened plate bending problem we prove that these higher order norms are bounded, although in principle these bounds could depend on $t$. We report a thorough numerical experimentation which gives numerical evidence that this is not the case, allowing us to assess the locking-free character of the method.

The outline of the paper is as follows. In Section 1 we present the mathematical setting of the load problem and prove existence and uniqueness. In Section 2 we analyze the concentrically stiffened plate and prove stability results, independent of the plate thickness for the in-plane and bending plate problems. In Section 3 we present the finite element discretizations of both problems, we obtain error estimates for the former and prove that the latter is well posed. In Section 4 we focus on the stiffened loading plate problem for which we prove error estimates. In Section 5 we report numerical results which allow us to assess the performance of the proposed method. First, to validate our code, we apply it to a vibration problem which was also solved in [12]. Secondly we apply the proposed method to a couple of problems whose limit behavior as the thickness becomes small is known. Finally we apply it to a family of problems which allow us to demonstrate that the method is locking-free.

\section{The PRoblem of the STIFFEned Plate}

We consider a plate entirely crossed by a rod which act as a stiffener. We restrict our attention to plates with constant thickness and rods with uniform cross section. If the mid-line of the rod lies on the mid-surface of the plate we call it a concentrically stiffened plate. Otherwise it is called an eccentrically stiffened plate (see Fig. 1). Most of this paper is devoted to concentrically stiffened plates except for the present section which is valid for eccentrically stiffened plates too.

We consider a coordinate system such that the plate occupies the domain $\Omega \times(-t / 2, t / 2)$, where $t>0$ is the plate thickness and $\Omega$ is the mid-surface of the plate, which is assumed to be polygonal. Moreover we assume that 

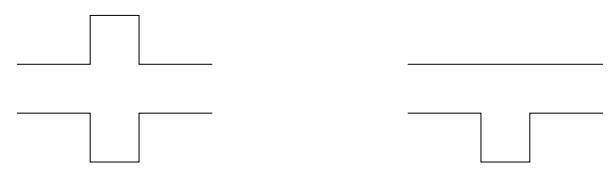

Figure 1. Transverse sections of concentrically (left) and eccentrically (right) stiffened plates.
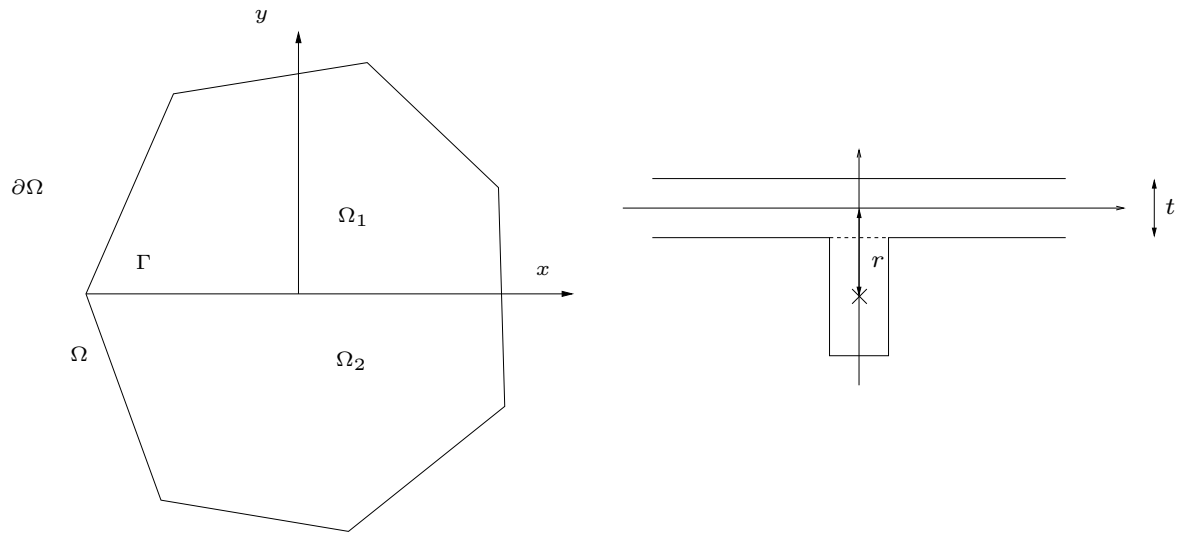

Figure 2. Geometry of a stiffened plate.

the coordinate system is such that the mid-line of the rod lies on the plane $y=0$. We denote by $r$ the distance between the rod mid-line and the plate mid-surface $(r=0$ for concentrically stiffened plates). The problem will be posed in the two dimensional domain $\Omega$. We denote $\Gamma:=\{(x, y) \in \Omega: y=0\}, \Omega_{1}:=\{(x, y) \in \Omega: y>0\}$ and $\Omega_{2}:=\{(x, y) \in \Omega: y<0\}$. We illustrate these definitions in Figure 2 .

We restrict our analysis to the case of a plate modeled by Reissner-Mindlin equations and completely clamped by its lateral boundary $\partial \Omega$. We denote by $\boldsymbol{u}=\left(u_{1}, u_{2}\right), w$ and $\boldsymbol{\beta}=\left(\beta_{1}, \beta_{2}\right)$ the mid-surface in-plane plate displacements, the transverse displacements and the rotations, respectively. In its turn, $u^{\mathrm{s}}, v^{\mathrm{s}}$, $w^{\mathrm{s}}$ denote the stiffener displacements in each coordinate direction and, $\theta_{x}^{\mathrm{s}}, \theta_{y}^{\mathrm{s}}$ and $\theta_{z}^{\mathrm{s}}$ the stiffener rotations with respect to each axis.

To derive the equations of the stiffened plate we will obtain first an expression for its total energy. With this aim, we add the corresponding energies of the plate and the stiffener. However, for the latter, the shear and bending terms in the plane of the plate are typically neglected (see [12]). We consider a load acting on the plate, and denote by $\boldsymbol{f}$ its in-plane component, and by $g$ the transverse one. Therefore, the total energy including the works of the loads reads

$$
\begin{aligned}
E_{t}:= & \frac{t}{2} a_{1}(\boldsymbol{u}, \boldsymbol{u})+\frac{t^{3}}{2} a_{2}(\boldsymbol{\beta}, \boldsymbol{\beta})+t \int_{\Omega} \kappa \mu(\nabla w-\boldsymbol{\beta})^{2}+\int_{\Gamma} E^{\mathrm{s}} A\left(\frac{\mathrm{d} u^{\mathrm{s}}}{\mathrm{d} x}\right)^{2}+\int_{\Gamma} E^{\mathrm{s}} I_{y y}\left(\frac{\mathrm{d} \theta_{y}^{\mathrm{s}}}{\mathrm{d} x}\right)^{2} \\
& +\int_{\Gamma} \mu^{\mathrm{s}} J\left(\frac{\mathrm{d} \theta_{x}^{\mathrm{s}}}{\mathrm{d} x}\right)^{2}+\int_{\Gamma} \kappa^{\mathrm{s}} \mu^{\mathrm{s}} A\left(\frac{\mathrm{d} w^{\mathrm{s}}}{\mathrm{d} x}+\theta_{y}^{\mathrm{s}}\right)^{2}-t \int_{\Omega} \boldsymbol{f} \cdot \boldsymbol{u}-\int_{\Omega} g w .
\end{aligned}
$$

In the above equation

$$
a_{1}(\boldsymbol{u}, \boldsymbol{v}):=\int_{\Omega} \frac{E \nu}{(1+\nu)(1-2 \nu)} \operatorname{div} \boldsymbol{u} \operatorname{div} \boldsymbol{v}+\int_{\Omega} \frac{E}{1+\nu} \boldsymbol{\epsilon}(\boldsymbol{u}): \boldsymbol{\epsilon}(\boldsymbol{v})
$$




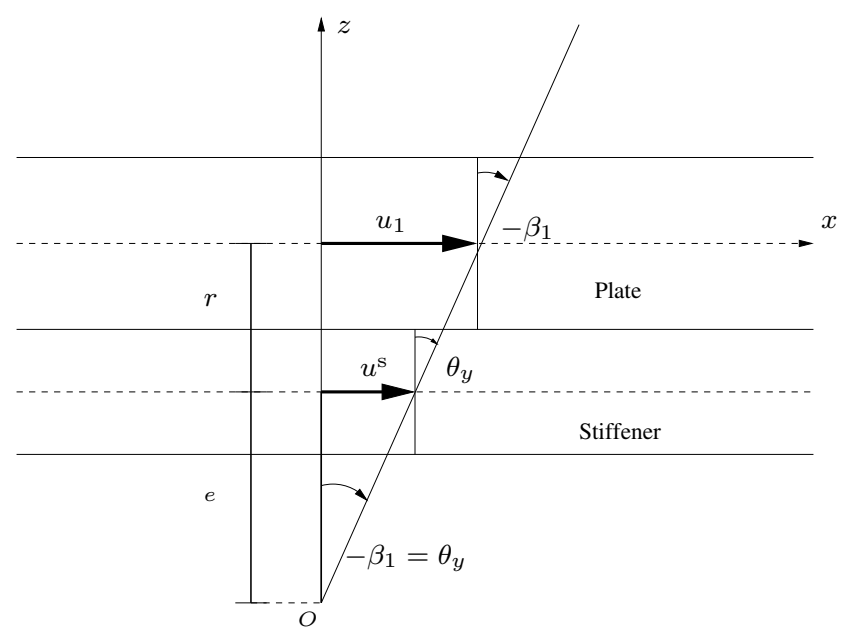

FIGURE 3. Geometric illustration of (1.5).

and

$$
a_{2}(\boldsymbol{\beta}, \boldsymbol{\eta}):=\frac{1}{12}\left\{\int_{\Omega} \frac{E \nu}{1-\nu^{2}} \operatorname{div} \boldsymbol{\beta} \operatorname{div} \boldsymbol{\eta}+\int_{\Omega} \frac{E}{1+\nu} \boldsymbol{\epsilon}(\boldsymbol{\beta}): \boldsymbol{\epsilon}(\boldsymbol{\eta})\right\}
$$

are the plane strain and plane stress bilinear forms, respectively. Above $\boldsymbol{\epsilon}(\boldsymbol{v}):=\frac{1}{2}\left(D \boldsymbol{v}+D \boldsymbol{v}^{t}\right)$ denotes the linearized strain tensor and the coefficients $E$ and $\nu$ are the Young and Poisson moduli. Moreover $\mu:=\frac{E}{2(1+\nu)}$ is the shear modulus of the plate. On the other hand, $A$ is the area of the stiffener section, $I_{y y}$ and $I_{z z}$ are the second moments of area with respect to $y$ - and $z$-axes, respectively, $J:=I_{y y}+I_{z z}, E^{\mathrm{s}}$ and $\nu^{\mathrm{s}}$ the Young and Poisson moduli of the stiffener and $\mu^{\mathrm{s}}:=\frac{E^{\mathrm{s}}}{2\left(1+\nu^{\mathrm{s}}\right)}$ the shear modulus. Finally $\kappa$ and $\kappa^{\mathrm{s}}$ are the shear correction factors for the plate and the rod, respectively. All the parameters are strictly positive constants. In the case of the stiffener this corresponds to a rod of arbitrary uniform section. Notice that, since the bending and shear energy of the stiffener have been neglected, the variables $v^{\mathrm{s}}$ and $\theta_{z}$ do not appear in the expression for the total energy.

The appropriate functional spaces for displacements and rotations are the following: $\boldsymbol{u}, \boldsymbol{\beta} \in H_{0}^{1}(\Omega)^{2} ; w \in$ $H_{0}^{1}(\Omega) ; u^{\mathrm{s}}, w^{\mathrm{s}}, \theta_{x}^{\mathrm{s}}, \theta_{y}^{\mathrm{s}} \in H_{0}^{1}(\Gamma)$. For the loads we consider $f \in L^{2}(\Omega)^{2}$ and $g \in L^{2}(\Omega)$. The stiffened plate problem is obtained by minimizing the total energy subject to the following kinematic constraints:

$$
\begin{aligned}
u_{1} & =u^{\mathrm{s}}-r \beta_{1}, \\
w & =w^{\mathrm{s}}, \\
\beta_{2} & =\theta_{x}^{\mathrm{s}}, \\
\beta_{1} & =-\theta_{y}^{\mathrm{s}} .
\end{aligned}
$$

The constraint (1.2) comes from the fact that the plate and the rod are joint monolithically. Moreover, the rotations of the plate and the stiffener are the same in this model, which leads to (1.3) and (1.4). Finally (1.1) can be easily deduced. In fact, using the notation from Figure 3, we have that

$$
\frac{u_{1}}{r+e}=\frac{u^{\mathrm{s}}}{e}
$$

Therefore approximating $-\beta_{1}$ by $\frac{u^{\mathrm{s}}}{e}$, straightforward computations lead to (1.1). 
For the analysis, we introduce these constraints by means of Lagrange multipliers as follows:

$$
\begin{aligned}
\int_{\Gamma} \lambda_{1}\left(u_{1}-u^{\mathrm{s}}+r \beta_{1}\right) & =0 \\
\int_{\Gamma} \lambda_{2}\left(w-w^{\mathrm{s}}\right) & =0 \\
\int_{\Gamma} \lambda_{3}\left(\beta_{2}-\theta_{x}^{\mathrm{s}}\right) & =0 \\
\int_{\Gamma} \lambda_{4}\left(\beta_{1}+\theta_{y}^{\mathrm{s}}\right) & =0
\end{aligned}
$$

In the expressions above and throughout the paper, to simplify the notation, we write

$$
\int_{\Gamma} \lambda \eta:=\langle\lambda, \eta\rangle_{H^{-1 / 2}(\Gamma) \times H_{00}^{1 / 2}(\Gamma)}
$$

where in this case $H_{00}^{1 / 2}(\Gamma):=\left\{\left.v\right|_{\Gamma}: v \in H_{0}^{1}(\Omega)\right\}$ endowed with the intrinsic norm of $H^{1 / 2}(\Gamma)($ see $[10])$ and $H^{-1 / 2}(\Gamma):=H_{00}^{1 / 2}(\Gamma)^{\prime}$, with $L^{2}(\Gamma)$ as pivot space.

Therefore, by minimizing $E_{t}$ subject to the constraints (1.1)-(1.4), we arrive at the following clamped stiffened plate problem:

Find $(\boldsymbol{U}, \boldsymbol{\lambda}) \in \mathcal{H} \times \mathcal{Q}$ such that

$$
\begin{aligned}
\mathscr{A}(\boldsymbol{U}, \boldsymbol{V})+\mathscr{B}(\boldsymbol{V}, \boldsymbol{\lambda}) & =\mathscr{F}(\boldsymbol{V}) \quad \forall \boldsymbol{V} \in \mathcal{H}, \\
\mathscr{B}(\boldsymbol{U}, \boldsymbol{\chi}) & =0 \quad \forall \chi \in \mathcal{Q},
\end{aligned}
$$

where

$$
\begin{aligned}
& \boldsymbol{U}:=\left(\boldsymbol{u}, \boldsymbol{\beta}, w, u^{\mathrm{s}}, w^{\mathrm{s}}, \theta_{x}^{\mathrm{s}}, \theta_{y}^{\mathrm{s}}\right), \quad \boldsymbol{V}:=\left(\boldsymbol{v}, \boldsymbol{\eta}, z, z^{\mathrm{s}}, v^{\mathrm{s}}, \varphi_{x}^{\mathrm{s}}, \varphi_{y}^{\mathrm{s}}\right) \in \mathcal{H}:=H_{0}^{1}(\Omega)^{2} \times H_{0}^{1}(\Omega)^{2} \times H_{0}^{1}(\Omega) \times H_{0}^{1}(\Gamma)^{4} \\
& \boldsymbol{\lambda}:=\left(\lambda_{1}, \lambda_{2}, \lambda_{3}, \lambda_{4}\right), \quad \chi:=\left(\chi_{1}, \chi_{2}, \chi_{3}, \chi_{4}\right) \in \mathcal{Q}:=H^{-1 / 2}(\Gamma)^{4} \\
& \mathscr{A}(\boldsymbol{U}, \boldsymbol{V}):= t a_{1}(\boldsymbol{u}, \boldsymbol{v})+t^{3} a_{2}(\boldsymbol{\beta}, \boldsymbol{\eta})+t \int_{\Omega} \kappa \mu(\nabla w-\boldsymbol{\beta}) \cdot(\nabla z-\boldsymbol{\eta})+\int_{\Gamma} E^{\mathrm{s}} A \frac{\mathrm{d} u^{\mathrm{s}}}{\mathrm{d} x} \frac{\mathrm{d} v^{\mathrm{s}}}{\mathrm{d} x} \\
&+\int_{\Gamma} \kappa^{\mathrm{s}} \mu^{\mathrm{s}} A\left(\frac{\mathrm{d} w^{\mathrm{s}}}{\mathrm{d} x}+\theta_{y}^{\mathrm{s}}\right)\left(\frac{\mathrm{d} z^{\mathrm{s}}}{\mathrm{d} x}+\varphi_{y}^{\mathrm{s}}\right)+\int_{\Gamma} E^{\mathrm{s}} I_{y y} \frac{\mathrm{d} \theta_{y}^{\mathrm{s}}}{\mathrm{d} x} \frac{\mathrm{d} \varphi_{y}^{\mathrm{s}}}{\mathrm{d} x}+\int_{\Gamma} \mu^{\mathrm{s}} J \frac{\mathrm{d} \theta_{x}^{\mathrm{s}}}{\mathrm{d} x} \frac{\mathrm{d} \varphi_{x}^{\mathrm{s}}}{\mathrm{d} x} \\
& \mathscr{B}(\boldsymbol{U}, \boldsymbol{\lambda}):= \int_{\Gamma} \lambda_{1}\left(u_{1}-u^{\mathrm{s}}+r \beta_{1}\right)+\int_{\Gamma} \lambda_{2}\left(w-w^{\mathrm{s}}\right)+\int_{\Gamma} \lambda_{3}\left(\beta_{2}-\theta_{x}^{\mathrm{s}}\right)+\int_{\Gamma} \lambda_{4}\left(\beta_{1}+\theta_{y}^{\mathrm{s}}\right) \\
& \mathscr{F}(\boldsymbol{V}):= t \int_{\Omega} \boldsymbol{f} \cdot \boldsymbol{v}+t \int_{\Omega} g z .
\end{aligned}
$$

It is well known that the standard finite element methods applied to plates or rods are subject to "numerical locking". This means that they lead to unacceptable poor results for thin structures, unless the mesh size is excessively small. The standard methodology to deal with locking-free methods is to consider a family of problems depending on a small parameter: the plate thickness $t$. A method will be locking-free if the error estimates do not deteriorate as the thickness becomes small. In our case, to avoid dealing with more than one parameter, we restrict our attention to a stiffener such that

$$
A=t^{2} \tilde{A}, \quad I_{y y}=t^{4} \tilde{I}_{y y} \quad \text { and } \quad J=t^{4} \tilde{J}
$$


where $\tilde{A}, \tilde{I}_{y y}$ and $\tilde{J}$ are constants. This corresponds to a rod with a section having width and height proportional to $t$.

The stiffening effect of such a rod tends to disappear as $t$ tends to zero. To avoid this and obtaining a proper limit problem, we assume that the physical parameters of the rod increase as $t$ tends to zero. More precisely we assume that

$$
E^{\mathrm{s}}=\frac{\tilde{E}^{\mathrm{s}}}{t}
$$

where $\tilde{E}^{\text {s }}$ is a fixed constant. See [6] for a similar choice in the case of Kirchhoff stiffened plates. Correspondingly we define $\tilde{\mu}^{\mathrm{s}}:=\frac{\tilde{E}^{\mathrm{s}}}{1+\nu^{\mathrm{s}}}$ so that $\mu^{\mathrm{s}}=\tilde{\mu}^{\mathrm{s}}$. Scalings (1.11) and (1.12) ensure that if the loads are scaled as usual for plates, then the solution of problem (1.9)-(1.10) attains a limit as $t$ tends to zero and that, in the limit problem, the rod remains as a stiffener. This will be clearly seen in the numerical examples of Section 5 , where different scalings of the physical parameters are considered. It is shown therein that (1.12) leads to a stiffened limit problem (Test 4) whereas other scalings lead either to a vanishing or to a perfectly rigid stiffener (Sect. 5.2). Using these scalings we write the bilinear form $\mathscr{A}$ as follows:

$$
\begin{aligned}
\mathscr{A}(\boldsymbol{U}, \boldsymbol{V})= & t a_{1}(\boldsymbol{u}, \boldsymbol{v})+t^{3} a_{2}(\boldsymbol{\beta}, \boldsymbol{\eta})+t \int_{\Omega} \kappa \mu(\nabla w-\boldsymbol{\beta}) \cdot(\nabla z-\boldsymbol{\eta})+t \int_{\Gamma} \tilde{E}^{\mathrm{s}} \tilde{A} \frac{\mathrm{d} u^{\mathrm{s}} \mathrm{d} x}{\mathrm{~d} v^{\mathrm{s}}} \\
& +t \int_{\Gamma} \kappa^{\mathrm{s}} \tilde{\mu}^{\mathrm{s}} \tilde{A}\left(\frac{\mathrm{d} w^{\mathrm{s}}}{\mathrm{d} x}+\theta_{y}^{\mathrm{s}}\right)\left(\frac{\mathrm{d} z^{\mathrm{s}}}{\mathrm{d} x}+\varphi_{y}^{\mathrm{s}}\right)+t^{3} \int_{\Gamma} \tilde{E}^{\mathrm{s}} \tilde{I}_{y y} \frac{\mathrm{d} \theta_{y}^{\mathrm{s}}}{\mathrm{d} x} \frac{\mathrm{d} \varphi_{y}^{\mathrm{s}}}{\mathrm{d} x}+t^{3} \int_{\Gamma} \tilde{\mu}^{\mathrm{s}} \tilde{J} \frac{\mathrm{d} \theta_{x}^{\mathrm{s}}}{\mathrm{d} x} \frac{\mathrm{d} \varphi_{x}^{\mathrm{s}}}{\mathrm{d} x} .
\end{aligned}
$$

We are interested in proving the existence and uniqueness of the solution to problem (1.9)-(1.10). For this purpose we will show the ellipticity of $\mathscr{A}(\cdot, \cdot)$ in the whole space $\mathcal{H}$ and the inf-sup condition for $\mathscr{B}(\cdot, \cdot)$ in appropriate spaces.

Throughout the paper $C$ will denote a strictly positive constant, not necessarily the same at each occurrence, but always independent of $t$ and the mesh-size $h$, which will be introduced in the next section.

For the existence and uniqueness, we consider the continuous problem (1.9)-(1.10) with $0<t \leq 1$ fixed. The bilinear form $\mathscr{A}(\cdot, \cdot)$ is elliptic in $\mathcal{H}$, with an ellipticity constant depending of $t$ :

$$
\mathscr{A}(\boldsymbol{V}, \boldsymbol{V}) \geq C\left\{t^{3}\left(\|\boldsymbol{\eta}\|_{1, \Omega}^{2}+\|z\|_{1, \Omega}^{2}+\left\|\varphi_{y}^{\mathrm{s}}\right\|_{1, \Omega}^{2}+\left\|z^{\mathrm{s}}\right\|_{1, \Omega}^{2}+\left\|\varphi_{x}^{\mathrm{s}}\right\|_{1, \Gamma}^{2}\right)+t\left(\|\boldsymbol{v}\|_{1, \Omega}^{2}+\left\|v^{\mathrm{s}}\right\|_{1, \Gamma}^{2}\right)\right\} .
$$

In fact, the inequality above follows from

$$
\begin{aligned}
& t a_{1}(\boldsymbol{v}, \boldsymbol{v}) \geq C t\|\boldsymbol{v}\|_{1, \Omega}^{2} \quad \forall \boldsymbol{v} \in H_{0}^{1}(\Omega)^{2}, \\
& a_{2}(\boldsymbol{\eta}, \boldsymbol{\eta}) \geq C\|\boldsymbol{\eta}\|_{1, \Omega}^{2} \quad \forall \boldsymbol{\eta} \in H_{0}^{1}(\Omega)^{2}, \\
& t^{3} a_{2}(\boldsymbol{\eta}, \boldsymbol{\eta})+t \int_{\Omega} \kappa \mu|\nabla z-\boldsymbol{\eta}|^{2} \geq C t^{3}\left\{\|\boldsymbol{\eta}\|_{1, \Omega}^{2}+\|z\|_{1, \Omega}^{2}\right\} \quad \forall \boldsymbol{\eta} \in H_{0}^{1}(\Omega)^{2}, z \in H_{0}^{1}(\Omega), \\
& t \int_{\Gamma} \tilde{E}^{\mathrm{s}} \tilde{A}\left(\frac{\mathrm{d} v^{\mathrm{s}}}{\mathrm{d} x}\right)^{2} \geq C t\left\|v^{\mathrm{s}}\right\|_{1, \Gamma}^{2} \quad \forall v^{\mathrm{s}} \in H_{0}^{1}(\Gamma), \\
& t^{3} \int_{\Gamma} \tilde{\mu}^{\mathrm{s}} \tilde{J}\left(\frac{\mathrm{d} \varphi_{x}^{\mathrm{s}}}{\mathrm{d} x}\right)^{2} \geq C t^{3}\left\|\varphi_{x}^{\mathrm{s}}\right\|_{1, \Gamma}^{2} \quad \forall \varphi_{x}^{\mathrm{s}} \in H_{0}^{1}(\Gamma), \\
& t^{3} \int_{\Gamma} \tilde{E}^{\mathrm{s}} \tilde{I}_{y y}\left(\frac{\mathrm{d} \varphi_{y}^{\mathrm{s}}}{\mathrm{d} x}\right)^{2}+t \int_{\Gamma} \kappa^{\mathrm{s}} \tilde{\mu}^{\mathrm{s}} \tilde{A}\left|\frac{\mathrm{d} z^{\mathrm{s}}}{\mathrm{d} x}+\varphi_{y}^{\mathrm{s}}\right|^{2} \geq C t^{3}\left\{\left\|z^{\mathrm{s}}\right\|_{1, \Gamma}^{2}+\left\|\varphi_{y}^{\mathrm{s}}\right\|_{1, \Gamma}^{2}\right\} \quad \forall \varphi_{y}^{\mathrm{s}}, z^{\mathrm{s}} \in H_{0}^{1}(\Gamma) .
\end{aligned}
$$

The inequalities (1.14) and (1.15) are consequences of Korn's inequalities, whereas (1.16) is a classical result, (see [5]). In its turn, (1.17)-(1.19) follow from the scalings (1.11)-(1.12), Poincaré inequality and a straightforward computation for $\int_{\Gamma}\left|\frac{\mathrm{d} z^{\mathrm{s}}}{\mathrm{d} x}+\varphi_{y}^{\mathrm{s}}\right|^{2}$ (see [1]). 
On the other hand, $\mathscr{B}(\cdot, \cdot)$ satisfies an inf-sup condition: There exists $C>0$, independent of $t$, such that,

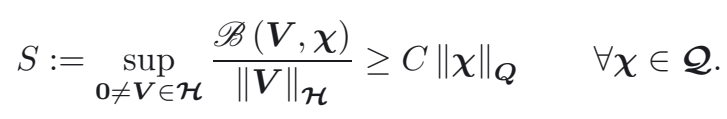

To prove this, we consider separately each component of $\boldsymbol{\chi}$. For the first one, we take $v_{1} \in H_{0}^{1}(\Omega)$ arbitrary and the remaining components of $\boldsymbol{V}$ equal to zero, to write

$$
S \geq \sup _{0 \neq v_{1} \in H_{0}^{1}(\Omega)} \frac{\int_{\Gamma} \chi_{1} v_{1}}{\left\|v_{1}\right\|_{1, \Omega}} \geq C_{1} \sup _{0 \neq \psi \in H_{00}^{1 / 2}(\Gamma)} \frac{\int_{\Gamma} \chi_{1} \psi}{\|\psi\|_{1 / 2, \Gamma}}=C\left\|\chi_{1}\right\|_{-1 / 2, \Gamma} .
$$

For the second inequality, we have used the equivalence between the intrinsic norm of $H_{00}^{1 / 2}(\Gamma)$ and the norm $\inf \left\{\|v\|_{1, \Omega}, v \in H_{0}^{1}(\Omega):\left.v\right|_{\Gamma}=\psi\right\}$ (see [10]). The same arguments allow us to prove that

$$
S \geq C\left\|\chi_{2}\right\|_{-1 / 2, \Gamma} \quad \text { and } \quad S \geq C\left\|\chi_{3}\right\|_{-1 / 2, \Gamma} .
$$

Finally, taking $\eta_{1} \in H_{0}^{1}(\Omega)$ arbitrary and the remaining components of $\boldsymbol{V}$ equal to zero and using (1.20), we have

$$
\left\|\chi_{4}\right\|_{-1 / 2, \Gamma} \leq C\left(S+r\left\|\chi_{1}\right\|_{-1 / 2, \Gamma}\right) \leq C(1+r) S
$$

which, since $r$ is bounded above, leads to

$$
S \geq C\left\|\chi_{4}\right\|_{-1 / 2, \Gamma} .
$$

The inequality above together with (1.21) and (1.20) allow us to prove the inf-sup condition for $\mathscr{B}$. This condition and the global ellipticity of $\mathscr{A}$ allow us to use the standard theory for mixed problems (see [5]) to conclude the following theorem:

Theorem 1.1. For any $t>0$ fixed, problem (1.9)-(1.10) has a unique solution $(\boldsymbol{U}, \boldsymbol{\lambda}) \in \mathcal{H} \times \mathcal{Q}$.

Remark 1.2. Solution to problem (1.9)-(1.10) is bounded in terms of $\|\mathscr{F}\|_{\mathcal{H}^{\prime}}$. However, in principle, this bound is not necessarily uniform with respect to $t$, since the ellipticity and continuity constants of the bilinear form $\mathscr{A}$ depend on different powers of the thickness (see [5], Prop. II.1.3).

\section{Concentrically stiffened Plates}

The analysis of the previous section is valid for concentrically as well as eccentrically stiffened plates. For the latter, in-plane and transverse terms are fully coupled and each one involve different scales with respect to the thickness. In this regard, this resembles what happens in the shell problem, for which the analysis is much less mature than for plates.

This is the reason why, from now on, we restrict our attention to concentrically stiffened plates (i.e., $r=0$, see Fig. 2). In this case, the plate problem decomposes into two uncoupled ones: the in-plane and the bending stiffened plate problems, as it happens for non stiffened plates. In fact, the in-plane displacements $\boldsymbol{u}$ and $u^{\mathrm{s}}$ relate with the remaining variables only through equation (1.5) which is part of (1.10) and this happen only if $r \neq 0$. For $r=0$ we are led to the following two uncoupled problems, the first one for the in-plane terms and the second one for the bending terms:

Find $\left(\boldsymbol{u}, u^{\mathrm{s}}\right) \in H_{0}^{1}(\Omega)^{2} \times H_{0}^{1}(\Gamma)$ and $\lambda_{1} \in H^{-1 / 2}(\Gamma)$ such that

$$
\begin{aligned}
t a_{1}(\boldsymbol{u}, \boldsymbol{v})+t \int_{\Gamma} \tilde{E}^{\mathrm{s}} \tilde{A} \frac{\mathrm{d} u^{\mathrm{s}}}{\mathrm{d} x} \frac{\mathrm{d} v^{\mathrm{s}}}{\mathrm{d} x}+\int_{\Gamma} \lambda_{1}\left(v_{1}-v^{\mathrm{s}}\right) & =t \int_{\Omega} \boldsymbol{f} \cdot \boldsymbol{v} \quad \forall\left(\boldsymbol{v}, v^{\mathrm{s}}\right) \in H_{0}^{1}(\Omega)^{2} \times H_{0}^{1}(\Gamma), \\
\int_{\Gamma} \chi_{1}\left(u_{1}-u^{\mathrm{s}}\right) & =0 \quad \forall \chi_{1} \in H^{-1 / 2}(\Gamma) .
\end{aligned}
$$


Find $\left(\boldsymbol{\beta}, w, w^{\mathrm{s}}, \theta_{x}^{\mathrm{s}}, \theta_{y}^{\mathrm{s}}\right) \in H_{0}^{1}(\Omega)^{2} \times H_{0}^{1}(\Omega) \times H_{0}^{1}(\Gamma)^{3}$ and $\left(\lambda_{2}, \lambda_{3}, \lambda_{4}\right) \in H^{-1 / 2}(\Gamma)^{3}$ such that

$$
\begin{gathered}
t^{3} a_{2}(\boldsymbol{\beta}, \boldsymbol{\eta})+t \int_{\Omega} \kappa \mu(\nabla w-\boldsymbol{\beta}) \cdot(\nabla z-\boldsymbol{\eta})+t^{3} \int_{\Gamma} \tilde{E}^{\mathrm{s}} \tilde{I}_{y y} \frac{\mathrm{d} \theta_{y}^{\mathrm{s}}}{\mathrm{d} x} \frac{\mathrm{d} \varphi_{y}^{\mathrm{s}}}{\mathrm{d} x}+t^{3} \int_{\Gamma} \tilde{\mu}^{\mathrm{s}} \tilde{J} \frac{\mathrm{d} \theta_{x}^{\mathrm{s}}}{\mathrm{d} x} \frac{\mathrm{d} \varphi_{x}^{\mathrm{s}}}{\mathrm{d} x} \\
+t \int_{\Gamma} \kappa^{\mathrm{s}} \tilde{\mu}^{\mathrm{s}} \tilde{A}\left(\frac{\mathrm{d} w^{\mathrm{s}}}{\mathrm{d} x}+\theta_{y}^{\mathrm{s}}\right)\left(\frac{\mathrm{d} z^{\mathrm{s}}}{\mathrm{d} x}+\varphi_{y}^{\mathrm{s}}\right)+\int_{\Gamma} \lambda_{2}\left(z-z^{\mathrm{s}}\right)+\int_{\Gamma} \lambda_{3}\left(\eta_{2}-\varphi_{x}^{\mathrm{s}}\right)+\int_{\Gamma} \lambda_{4}\left(\eta_{1}+\varphi_{y}^{\mathrm{s}}\right)=t \int_{\Omega} g z \\
\forall\left(\boldsymbol{\eta}, z, z^{\mathrm{s}}, \varphi_{x}^{\mathrm{s}}, \varphi_{y}^{\mathrm{s}}\right) \in H_{0}^{1}(\Omega)^{2} \times H_{0}^{1}(\Omega) \times H_{0}^{1}(\Gamma)^{3} \\
\int_{\Gamma} \chi_{2}\left(w-w^{\mathrm{s}}\right)+\int_{\Gamma} \chi_{3}\left(\beta_{2}-\theta_{x}^{\mathrm{s}}\right)+\int_{\Gamma} \chi_{4}\left(\beta_{1}+\theta_{y}^{\mathrm{s}}\right)=0 \quad \forall\left(\chi_{2}, \chi_{3}, \chi_{4}\right) \in H^{-1 / 2}(\Gamma)^{3}
\end{gathered}
$$

From now on, to simplify a bit the notation, we set all the following constants equal to one: $\tilde{A}, \tilde{I}_{y y}, \tilde{J}, \kappa, \kappa_{\mathrm{s}}$, $\tilde{E}^{\mathrm{s}}, \tilde{\mu}^{\mathrm{s}}$, as well as $E$ (which appears in the definition of $a_{1}$ and $a_{2}$ ). Moreover, we consider values of $t$ ranging in $(0,1]$. Since these constants have been assumed to be independent of $t$, this does not affect any subsequent asymptotic analysis. Moreover to obtain a family of problems uniformly stable with respect to $t$, we consider a transverse load proportional to $t^{2}$, namely

$$
g=\tilde{g} t^{2}
$$

with $\tilde{g}$ independent of $t$. Finally, it is also convenient for the analysis to rescale the Lagrange multipliers according to the scales of the different problems in which they appear:

$$
\lambda_{1}=\tilde{\lambda}_{1} t \quad \text { and } \quad \lambda_{i}=\tilde{\lambda}_{i} t^{3}, i=2,3,4 .
$$

In what follows we analyze the resulting rescaled problems.

\subsection{Stiffened in-plane plate problem}

Using the scaling (2.6) in (2.1)-(2.2) and setting the constants equal to one, we obtain the following rescaled problem, in which we omit the tildes in $\tilde{\lambda}_{1}$ to simplify the notation:

Find $\left(\boldsymbol{u}, u^{\mathrm{s}}\right) \in H_{0}^{1}(\Omega)^{2} \times H_{0}^{1}(\Gamma)$ and $\lambda_{1} \in H^{-1 / 2}(\Gamma)$ such that

$$
\begin{aligned}
a_{1}(\boldsymbol{u}, \boldsymbol{v})+\int_{\Gamma} \frac{\mathrm{d} u^{\mathrm{s}}}{\mathrm{d} x} \frac{\mathrm{d} v^{\mathrm{s}}}{\mathrm{d} x}+\int_{\Gamma} \lambda_{1}\left(v_{1}-v^{\mathrm{s}}\right) & =\int_{\Omega} \boldsymbol{f} \cdot \boldsymbol{v} \quad \forall\left(\boldsymbol{v}, v^{\mathrm{s}}\right) \in H_{0}^{1}(\Omega)^{2} \times H_{0}^{1}(\Gamma), \\
\int_{\Gamma} \chi_{1}\left(u_{1}-u^{\mathrm{s}}\right) & =0 \quad \forall \chi_{1} \in H^{-1 / 2}(\Gamma) .
\end{aligned}
$$

This is a well posed problem completely independent of the thickness $t$. In fact, all the bilinear forms in this problem are continuous and the following ellipticity result holds:

$$
a_{1}(\boldsymbol{v}, \boldsymbol{v})+\int_{\Gamma}\left(\frac{\mathrm{d} v^{\mathrm{s}}}{\mathrm{d} x}\right)^{2} \geq C\left\{\|\boldsymbol{v}\|_{1, \Omega}^{2}+\left\|v^{\mathrm{s}}\right\|_{1, \Gamma}^{2}\right\} \quad \forall\left(\boldsymbol{v}, v^{\mathrm{s}}\right) \in H_{0}^{1}(\Gamma) \times H_{0}^{1}(\Omega)^{2} .
$$

Moreover, the arguments leading to (1.20) show that there exists $C>0$, independent of $t$, such that

$$
\sup _{0 \neq\left(\boldsymbol{v}, v^{\mathrm{s}}\right) \in H_{0}^{1}(\Omega)^{2} \times H_{0}^{1}(\Gamma)} \frac{\int_{\Gamma} \chi_{1}\left(v_{1}-v^{\mathrm{s}}\right)}{\|\boldsymbol{v}\|_{1, \Omega}+\left\|v^{\mathrm{s}}\right\|_{1, \Gamma}} \geq C\left\|\chi_{1}\right\|_{-1 / 2, \Gamma} \quad \forall \chi_{1} \in H^{-1 / 2}(\Gamma) .
$$

Thus, using Babuška-Brezzi theory (see [5]), we have the a-priori estimate

$$
\|\boldsymbol{u}\|_{1, \Omega}+\left\|u^{\mathrm{s}}\right\|_{1, \Gamma}+\left\|\lambda_{1}\right\|_{-1 / 2, \Gamma} \leq C\|f\|_{0, \Omega} .
$$




\subsection{Stiffened bending plate problem}

In this case we use the scalings (2.5) and (2.6) in (2.3)-(2.4). Thus if we denote

$$
\boldsymbol{H}:=H_{0}^{1}(\Omega)^{2} \times H_{0}^{1}(\Omega) \times H_{0}^{1}(\Gamma)^{3} \quad \text { and } \quad \boldsymbol{Q}:=H^{-1 / 2}(\Gamma)^{3},
$$

and set the constants equal to one again, then we arrive at the following rescaled problem, in which we omit the tildes in $\tilde{\lambda}_{i}, i=2,3,4$, and $\tilde{g}$ :

Find $\left(\boldsymbol{\beta}, w, w^{\mathrm{s}}, \theta_{x}^{\mathrm{s}}, \theta_{y}^{\mathrm{s}}\right) \in \boldsymbol{H}$ and $\left(\lambda_{2}, \lambda_{3}, \lambda_{4}\right) \in \boldsymbol{Q}$ such that

$$
\begin{gathered}
a_{2}(\boldsymbol{\beta}, \boldsymbol{\eta})+\frac{1}{t^{2}} \int_{\Omega}(\nabla w-\boldsymbol{\beta}) \cdot(\nabla z-\boldsymbol{\eta})+\int_{\Gamma} \frac{\mathrm{d} \theta_{x}^{\mathrm{s}}}{\mathrm{d} x} \frac{\mathrm{d} \varphi_{x}^{\mathrm{s}}}{\mathrm{d} x}+\int_{\Gamma} \frac{\mathrm{d} \theta_{y}^{\mathrm{s}}}{\mathrm{d} x} \frac{\mathrm{d} \varphi_{y}^{\mathrm{s}}}{\mathrm{d} x}+\frac{1}{t^{2}} \int_{\Gamma}\left(\frac{\mathrm{d} w^{\mathrm{s}}}{\mathrm{d} x}+\theta_{y}^{\mathrm{s}}\right)\left(\frac{\mathrm{d} z^{\mathrm{s}}}{\mathrm{d} x}+\varphi_{y}^{\mathrm{s}}\right) \\
+\int_{\Gamma} \lambda_{2}\left(z-z^{\mathrm{s}}\right)+\int_{\Gamma} \lambda_{3}\left(\eta_{2}-\varphi_{x}^{\mathrm{s}}\right)+\int_{\Gamma} \lambda_{4}\left(\eta_{1}+\varphi_{y}^{\mathrm{s}}\right)=\int_{\Omega} g z \quad \forall\left(\boldsymbol{\eta}, z, z^{\mathrm{s}}, \varphi_{x}^{\mathrm{s}}, \varphi_{y}^{\mathrm{s}}\right) \in \boldsymbol{H}, \\
\int_{\Gamma} \chi_{2}\left(w-w^{\mathrm{s}}\right)+\int_{\Gamma} \chi_{3}\left(\beta_{2}-\theta_{x}^{\mathrm{s}}\right)+\int_{\Gamma} \chi_{4}\left(\beta_{1}+\theta_{y}^{\mathrm{s}}\right)=0 \quad \forall\left(\chi_{2}, \chi_{3}, \chi_{4}\right) \in \boldsymbol{Q} .
\end{gathered}
$$

This problem has a unique solution. In fact, this is a consequence of Theorem 1.1 and the equivalence of problem (1.9)-(1.10) with (2.7)-(2.8) and (2.10)-(2.11) in the case $r=0$. However, this theorem does not imply that the solution is bounded independently of $t$ (cf. Rem. 1.2). Our next goal is to prove that, for a non vanishing $g$, the solution of problem (2.10)-(2.11) is bounded above and below far from zero, both uniformly with respect to $t$. More precisely, we will prove that for $g \neq 0$, there exist strictly positive constants $C_{1}$ and $C_{2}$ such that the solution of this problem satisfies

$$
C_{1} \leq\|\boldsymbol{\beta}\|_{1, \Omega}+\|w\|_{1, \Omega}+\left\|w^{\mathrm{s}}\right\|_{1, \Gamma}+\left\|\theta_{x}^{\mathrm{s}}\right\|_{1, \Gamma}+\left\|\theta_{y}^{\mathrm{s}}\right\|_{1, \Gamma} \leq C_{2},
$$

for all $t \in(0,1]$.

With this aim we consider the space

$$
\boldsymbol{W}:=\left\{(\boldsymbol{\eta}, z) \in H_{0}^{1}(\Omega)^{2} \times H_{0}^{1}(\Omega): \frac{\mathrm{d} \eta_{1}}{\mathrm{~d} x}, \frac{\mathrm{d} \eta_{2}}{\mathrm{~d} x}, \frac{\mathrm{d} z}{\mathrm{~d} x} \in L^{2}(\Gamma)\right\},
$$

endowed with the norm

$$
\|(\boldsymbol{\eta}, z)\|_{\boldsymbol{W}}^{2}:=\|\boldsymbol{\eta}\|_{1, \Omega}^{2}+\|z\|_{1, \Omega}^{2}+\left\|\eta_{1}\right\|_{1, \Gamma}^{2}+\left\|\eta_{2}\right\|_{1, \Gamma}^{2}+\|z\|_{1, \Gamma}^{2}, \quad(\boldsymbol{\eta}, z) \in \boldsymbol{W} .
$$

Equation (2.11) is equivalent to.

$$
w^{\mathrm{s}}=w, \quad \theta_{x}^{\mathrm{s}}=\beta_{2} \quad \text { and } \quad \theta_{y}^{\mathrm{s}}=-\beta_{1} \quad \text { on } \Gamma,
$$

which in its turn implies that $(\boldsymbol{\beta}, w) \in \boldsymbol{W}$. Using this and testing (2.10) with functions satisfying the same constraints, we arrive at

$$
\begin{aligned}
& a_{2}(\boldsymbol{\beta}, \boldsymbol{\eta})+\frac{1}{t^{2}} \int_{\Omega}(\nabla w-\boldsymbol{\beta}) \cdot(\nabla z-\boldsymbol{\eta})+\int_{\Gamma} \frac{\partial \beta_{1}}{\partial x} \frac{\partial \eta_{1}}{\partial x}+\int_{\Gamma} \frac{\partial \beta_{2}}{\partial x} \frac{\partial \eta_{2}}{\partial x}+\frac{1}{t^{2}} \int_{\Gamma}\left(\frac{\partial w}{\partial x}-\beta_{1}\right)\left(\frac{\partial z}{\partial x}-\eta_{1}\right)=\int_{\Omega} g z \\
& \forall(\boldsymbol{\eta}, z) \in \boldsymbol{W} .
\end{aligned}
$$

Testing the above problem with $(\eta, z)=(\boldsymbol{\beta}, w)$ we obtain

$$
a_{2}(\boldsymbol{\beta}, \boldsymbol{\beta})+\frac{1}{t^{2}} \int_{\Omega}(\nabla w-\boldsymbol{\beta})^{2}+\int_{\Gamma}\left(\frac{\partial \beta_{1}}{\partial x}\right)^{2}+\int_{\Gamma}\left(\frac{\partial \beta_{1}}{\partial x}\right)^{2}+\frac{1}{t^{2}} \int_{\Gamma}\left(\frac{\partial w}{\partial x}-\beta_{1}\right)^{2}=\int_{\Omega} g w .
$$


On the other hand, from (1.16) we have that

$$
\|\boldsymbol{\beta}\|_{1, \Omega}^{2}+\|w\|_{1, \Omega}^{2} \leq C\left\{\frac{1}{t^{2}}\|\nabla w-\boldsymbol{\beta}\|_{0, \Omega}^{2}+a_{2}(\boldsymbol{\beta}, \boldsymbol{\beta})\right\},
$$

whereas from (1.17) and (1.19) we have

$$
\|w\|_{1, \Gamma}^{2}+\left\|\beta_{1}\right\|_{1, \Gamma}^{2}+\left\|\beta_{2}\right\|_{1, \Gamma}^{2} \leq C\left\{\frac{1}{t^{2}}\left\|\frac{\partial w}{\partial x}-\beta_{1}\right\|_{0, \Gamma}^{2}+\int_{\Gamma}\left(\frac{\partial \beta_{1}}{\partial x}\right)^{2}+\int_{\Gamma}\left(\frac{\partial \beta_{2}}{\partial x}\right)^{2}\right\} .
$$

Therefore, adding (2.16) and (2.17) and using (2.15), we obtain

$$
\|\boldsymbol{\beta}\|_{1, \Omega}^{2}+\|w\|_{1, \Omega}^{2}+\|w\|_{1, \Gamma}^{2}+\left\|\beta_{1}\right\|_{1, \Gamma}^{2}+\left\|\beta_{2}\right\|_{1, \Gamma}^{2} \leq C \int_{\Omega} g w,
$$

from which it follows that

$$
\|\boldsymbol{\beta}\|_{1, \Omega}+\|w\|_{1, \Omega}+\|w\|_{1, \Gamma}+\left\|\beta_{1}\right\|_{1, \Gamma}+\left\|\beta_{2}\right\|_{1, \Gamma} \leq C_{2}
$$

with $C_{2}$ only depending on $g$ and the domain $\Omega$.

To prove the other estimate in (2.12), we observe that the solution $(\boldsymbol{\beta}, w) \in \boldsymbol{W}$ to problem (2.14) is the minimum in $\boldsymbol{W}$ of the energy functional $E_{t}(\eta, z)$ defined by

$$
E_{t}(\eta, z):=\frac{1}{2}\left\{a_{2}(\boldsymbol{\eta}, \boldsymbol{\eta})+\frac{1}{t^{2}} \int_{\Omega}(\nabla z-\boldsymbol{\eta})^{2}+\int_{\Gamma}\left(\frac{\partial \eta_{1}}{\partial x}\right)^{2}+\int_{\Gamma}\left(\frac{\partial \eta_{2}}{\partial x}\right)^{2}+\frac{1}{t^{2}} \int_{\Gamma}\left(\frac{\partial z}{\partial x}-\eta_{1}\right)^{2}\right\}-\int_{\Omega} g z .
$$

Consider the following closed subspace of $\boldsymbol{W}$ :

$$
\boldsymbol{W}_{0}:=\{(\boldsymbol{\eta}, z) \in \boldsymbol{W}: \nabla z=\boldsymbol{\eta}\}=\left\{(\nabla z, z), z \in H_{0}^{2}(\Omega): \frac{\partial^{2} z}{\partial x \partial y}, \frac{\partial^{2} z}{\partial x^{2}} \in L^{2}(\Gamma)\right\} .
$$

For $(\boldsymbol{\eta}, z) \in \boldsymbol{W}_{0}$, the associated energy reads

$$
E_{t}(\eta, z):=\frac{1}{2}\left\{a_{2}(\nabla z, \nabla z)+\int_{\Gamma}\left(\frac{\partial^{2} z}{\partial x^{2}}\right)^{2}+\int_{\Gamma}\left(\frac{\partial^{2} z}{\partial x \partial y}\right)^{2}\right\}-\int_{\Omega} g z .
$$

We note that

$$
\min _{\boldsymbol{W}} E_{t} \leq \min _{\boldsymbol{W}_{0}} E_{t}<0 .
$$

In fact, the first inequality is clear, whereas for the second one we observe that the minimum of $E_{t}$ in $\boldsymbol{W}_{0}$ is attained at $(\eta, z)=\left(\nabla z_{0}, z_{0}\right) \in \boldsymbol{W}_{0}$ with $z_{0}$ satisfying

$$
a_{2}\left(\nabla z_{0}, \nabla z\right)+\int_{\Gamma} \frac{\partial^{2} z_{0}}{\partial x^{2}} \frac{\partial^{2} z}{\partial x^{2}}+\int_{\Gamma} \frac{\partial^{2} z_{0}}{\partial x \partial y} \frac{\partial^{2} z}{\partial x \partial y}=\int_{\Omega} g z \quad \forall(\nabla z, z) \in \boldsymbol{W}_{0} .
$$

The bilinear form on the left hand side is bounded and elliptic in $\boldsymbol{W}_{0}$, the latter because of (1.15). Hence, from the Lax-Milgram Lemma, there exists a unique solution of $(2.21)\left(\nabla z_{0}, z_{0}\right) \in \boldsymbol{W}_{0}$ and it satisfies $\left\|\left(\nabla z_{0}, z_{0}\right)\right\|_{\boldsymbol{W}}$ $\leq C\|g\|_{0, \Omega}$. Therefore, for $g \neq 0$, replacing (2.21) in (2.20), we have

$$
\min _{(\boldsymbol{\eta}, z) \in \boldsymbol{W}} E_{t}(\boldsymbol{\eta}, z) \leq \min _{(\boldsymbol{\eta}, z) \in \boldsymbol{W}_{0}} E_{t}(\boldsymbol{\eta}, z)=-\frac{1}{2}\left\{a_{2}\left(\nabla z_{0}, \nabla z_{0}\right)+\int_{\Gamma}\left(\frac{\partial^{2} z_{0}}{\partial x^{2}}\right)^{2}+\int_{\Gamma}\left(\frac{\partial^{2} z_{0}}{\partial x \partial y}\right)^{2}\right\}=:-C_{0}<0 .
$$


Since $\min _{\boldsymbol{W}} E_{t}(\boldsymbol{\eta}, z)$ is attained in the solution $(\boldsymbol{\beta}, w)$ of problem (2.14), we use this equation in (2.19) and (2.22) to write

$$
a_{2}(\boldsymbol{\beta}, \boldsymbol{\beta})+\frac{1}{t^{2}} \int_{\Omega}(\nabla w-\boldsymbol{\beta})^{2}+\int_{\Gamma}\left(\frac{\partial \beta_{1}}{\partial x}\right)^{2}+\int_{\Gamma}\left(\frac{\partial \beta_{2}}{\partial x}\right)^{2}+\frac{1}{t^{2}} \int_{\Gamma}\left(\frac{\partial w}{\partial x}-\beta_{1}\right)^{2} \geq 2 C_{0}
$$

On the other hand, testing $(2.14)$ with $(\boldsymbol{\eta}, z)=(\beta, w)$, we have

$$
\begin{aligned}
\frac{1}{t^{2}} \int_{\Omega}(\nabla w-\boldsymbol{\beta})^{2}+\frac{1}{t^{2}} \int_{\Gamma}\left(\frac{\partial w}{\partial x}-\beta_{1}\right)^{2} & =\int_{\Omega} g w-a_{2}(\boldsymbol{\beta}, \boldsymbol{\beta})-\int_{\Gamma}\left(\frac{\partial \beta_{1}}{\partial x}\right)^{2}-\int_{\Gamma}\left(\frac{\partial \beta_{2}}{\partial x}\right)^{2} \\
& \leq \int_{\Omega} g w \leq \frac{\epsilon}{2}\|g\|_{0, \Omega}^{2}+\frac{1}{2 \epsilon}\|w\|_{0, \Omega}^{2} \quad \forall \epsilon>0 .
\end{aligned}
$$

Substituting (2.24) in (2.23), choosing an adequate value of $\epsilon$ and using the boundedness of $a_{2}(\cdot, \cdot)$, we arrive at

$$
\|\boldsymbol{\beta}\|_{1, \Omega}+\|w\|_{1, \Omega}+\|w\|_{1, \Gamma}+\left\|\beta_{1}\right\|_{1, \Gamma}+\left\|\beta_{2}\right\|_{1, \Gamma} \geq C_{1}
$$

Therefore, recalling (2.13), we obtain the following result as a consequence of (2.18) and (2.25)

Proposition 2.1. Given a non vanishing $g \in L^{2}(\Omega)$, let $\left(\boldsymbol{\beta}, w, w^{\mathrm{s}}, \theta_{x}^{\mathrm{s}}, \theta_{y}^{\mathrm{s}}\right) \in \boldsymbol{H}$ be the solution of $(2.10)-(2.11)$. Then, there exists strictly positive constants $C_{1}, C_{2}$ independent of $t \in(0,1]$ such that

$$
C_{1} \leq\|\boldsymbol{\beta}\|_{1, \Omega}+\|w\|_{1, \Omega}+\left\|w^{\mathrm{s}}\right\|_{1, \Gamma}+\left\|\theta_{x}^{\mathrm{s}}\right\|_{1, \Gamma}+\left\|\theta_{y}^{\mathrm{s}}\right\|_{1, \Gamma} \leq C_{2} .
$$

In what follows we present some regularity results associated to the stiffened bending plate problem. First, we define the shear terms in the plate and the stiffener as follows:

$$
\begin{aligned}
\gamma & :=\frac{1}{t^{2}}(\nabla w-\beta), \\
\alpha & :=\frac{1}{t^{2}}\left(\frac{\mathrm{d} w^{\mathrm{s}}}{\mathrm{d} x}+\theta_{y}^{\mathrm{s}}\right) .
\end{aligned}
$$

Proposition 2.2. The solution of (2.10)-(2.11) satisfies $\left.\boldsymbol{\beta}\right|_{\Omega_{i}} \in H^{2}\left(\Omega_{i}\right)^{2},\left.w\right|_{\Omega_{i}} \in H^{2}\left(\Omega_{i}\right)^{2}, i=1,2$, and $\theta_{x}^{\mathrm{s}}$, $\theta_{y}^{\mathrm{s}}, w^{\mathrm{s}} \in H^{5 / 2}(\Gamma)$. Moreover, $\left.\gamma\right|_{\Omega_{i}} \in H^{1}\left(\Omega_{i}\right)^{2}, i=1,2$, and $\alpha \in H^{3 / 2}(\Gamma)$.

Proof. Testing (2.10) with $\varphi_{x}^{\mathrm{s}} \in H_{0}^{1}(\Gamma)$ and setting to zero all the other test functions, we have

$$
-\frac{\mathrm{d}^{2} \theta_{x}^{\mathrm{s}}}{\mathrm{d} x^{2}}=\lambda_{3} \in H^{-1 / 2}(\Gamma)
$$

and hence $\theta_{x}^{\mathrm{s}} \in H^{3 / 2}(\Gamma)$. In a similar way, testing (2.10) with $z^{\mathrm{s}} \neq 0$ and $\varphi_{y}^{\mathrm{s}} \neq 0$, we obtain that $\alpha \in H^{1 / 2}(\Gamma)$ and $\theta_{y}^{\mathrm{s}} \in H^{3 / 2}(\Gamma)$, respectively. Consequently, (2.27) yields $w^{\mathrm{s}} \in H^{3 / 2}(\Gamma)$. Next we extend $w^{\mathrm{s}}$ to the whole domain $\Omega$ in such a way that the extension belongs to $H^{2}$ of each subdomain $\Omega_{i}$. More precisely, let $w_{0} \in H_{0}^{1}(\Omega)$ be such that $\left.w_{0}\right|_{\Omega_{i}} \in H^{2}\left(\Omega_{i}\right), i=1,2$, (see [11], Thm. 1.5.2.8) and

$$
\left.w_{0}\right|_{\Gamma}=w^{\mathrm{s}} .
$$

Analogously, let $\boldsymbol{\beta}_{0} \in H_{0}^{1}(\Omega)^{2}:\left.\boldsymbol{\beta}_{0}\right|_{\Omega_{i}} \in H^{2}\left(\Omega_{i}\right), i=1,2$, and

$$
\left.\boldsymbol{\beta}_{0}\right|_{\Gamma}=\left(-\theta_{y}^{\mathrm{s}}, \theta_{x}^{\mathrm{s}}\right) .
$$


Let $\tilde{\boldsymbol{\beta}} \in H_{0}^{1}(\Omega)^{2}$ and $\tilde{w} \in H_{0}^{1}(\Omega)$ be defined by

$$
\boldsymbol{\beta}=\tilde{\boldsymbol{\beta}}+\boldsymbol{\beta}_{0} \quad \text { and } \quad w=\tilde{w}+w_{0} .
$$

Since $\left.\boldsymbol{\beta}\right|_{\Gamma}=\left.\boldsymbol{\beta}_{0}\right|_{\Gamma}$ and $\left.w\right|_{\Gamma}=\left.w_{0}\right|_{\Gamma}$, we have that $\left.\tilde{\boldsymbol{\beta}}\right|_{\Omega_{i}} \in H_{0}^{1}\left(\Omega_{i}\right)^{2}$ and $\left.\tilde{w}\right|_{\Omega_{i}} \in H_{0}^{1}\left(\Omega_{i}\right), i=1,2$. Our next goal is to show that $\left(\left.\tilde{\boldsymbol{\beta}}\right|_{\Omega_{i}},\left.\tilde{w}\right|_{\Omega_{i}}\right)$ is the solution of a plate problem for which an additional regularity result holds. With this aim, we take $\boldsymbol{\eta} \in H_{0}^{1}\left(\Omega_{i}\right)^{2}$ and $z \in H_{0}^{1}\left(\Omega_{i}\right)$ and extend them by zero to $\Omega$. These extensions, that we also denote $\boldsymbol{\eta}$ and $z$, clearly satisfy $\boldsymbol{\eta} \in H_{0}^{1}(\Omega)^{2}$ and $z \in H_{0}^{1}(\Omega)$. If we take such $\boldsymbol{\eta}$ and $z$ with vanishing $z^{\mathrm{s}}$, $\varphi_{x}^{\mathrm{s}}$ and $\varphi_{y}^{\mathrm{s}}$ in $(2.10)$, we obtain that

$$
a_{2}^{i}(\boldsymbol{\beta}, \boldsymbol{\eta})+\frac{1}{t^{2}} \int_{\Omega_{i}}(\nabla w-\boldsymbol{\beta}) \cdot(\nabla z-\boldsymbol{\eta})=\int_{\Omega_{i}} g z \quad \forall(\boldsymbol{\eta}, z) \in H_{0}^{1}\left(\Omega_{i}\right)^{2} \times H_{0}^{1}\left(\Omega_{i}\right),
$$

where

$$
a_{2}^{i}(\boldsymbol{\beta}, \boldsymbol{\eta}):=\frac{1}{12}\left\{\int_{\Omega_{i}} \frac{E \nu}{1-\nu^{2}} \operatorname{div} \boldsymbol{\beta} \operatorname{div} \boldsymbol{\eta}+\int_{\Omega_{i}} \frac{E}{1+\nu} \epsilon(\boldsymbol{\beta}): \boldsymbol{\epsilon}(\boldsymbol{\eta})\right\} .
$$

Therefore, using (2.29), we have that $\left.\tilde{\boldsymbol{\beta}}\right|_{\Omega_{i}} \in H_{0}^{1}\left(\Omega_{i}\right)^{2}$ and $\left.\tilde{w}\right|_{\Omega_{i}} \in H_{0}^{1}\left(\Omega_{i}\right)$ solve the following problem:

$$
\begin{aligned}
a_{2}^{i}(\tilde{\boldsymbol{\beta}}, \boldsymbol{\eta})+\frac{1}{t^{2}} \int_{\Omega_{i}}(\nabla \tilde{w}-\tilde{\boldsymbol{\beta}}) \cdot(\nabla z-\boldsymbol{\eta}) & =-a_{2}^{i}\left(\boldsymbol{\beta}_{0}, \boldsymbol{\eta}\right)-\frac{1}{t^{2}} \int_{\Omega_{i}}\left(\nabla w_{0}-\boldsymbol{\beta}_{0}\right) \cdot(\nabla z-\boldsymbol{\eta})+\int_{\Omega_{i}} g z \\
& =: \int_{\Omega_{i}} \boldsymbol{F} \cdot \boldsymbol{\eta}+\int_{\Omega_{i}} G z \quad \forall(\boldsymbol{\eta}, z) \in H_{0}^{1}\left(\Omega_{i}\right)^{2} \times H_{0}^{1}\left(\Omega_{i}\right) .
\end{aligned}
$$

Notice that $\boldsymbol{F} \in L^{2}(\Omega)^{2}$ and $G \in L^{2}(\Omega)$, because $\left.\boldsymbol{\beta}_{0}\right|_{\Omega_{i}} \in H^{2}\left(\Omega_{i}\right)^{2}$ and $\left.w_{0}\right|_{\Omega_{i}} \in H^{2}\left(\Omega_{i}\right)$. Hence, using the regularity results of [2], Theorem 2.1, we have that $(\tilde{\boldsymbol{\beta}}, \tilde{w}) \in H^{2}\left(\Omega_{i}\right)^{2} \times H^{2}\left(\Omega_{i}\right)$, which using (2.29) yields the regularity for $\boldsymbol{\beta}$.

The next step is to prove additional regularity for $\gamma$ and $\alpha$. With this aim, we observe first that by using the definition of $\boldsymbol{\gamma}$ in (2.26) and the regularity of $\boldsymbol{\beta}$ and $w$ we have that $\left.\gamma\right|_{\Omega_{i}} \in H^{1}\left(\Omega_{i}\right)^{2}, i=1,2$.

On the other hand, testing problem $(2.30)$ with $z \in H_{0}^{1}\left(\Omega_{i}\right)$ and $\boldsymbol{\eta}=\mathbf{0}$, we obtain that

$$
-\operatorname{div} \gamma=g \quad \text { in } \Omega_{i}
$$

Therefore, testing (2.10) with $z \in H_{0}^{1}(\Omega)$ and all the other variables set to zero, integrating by parts in each subdomain $\Omega_{i}$ and using the equation above, we have that

$$
\int_{\Gamma} \llbracket \gamma \cdot \boldsymbol{n} \rrbracket z+\int_{\Gamma} \lambda_{2} z=0
$$

where $\llbracket \cdot \rrbracket$ denotes the jump across $\Gamma$. Consequently, from the regularity of $\gamma$, we obtain $\lambda_{2} \in H^{1 / 2}(\Gamma)$. Therefore, testing (2.10) with $z^{\mathrm{s}} \in H_{0}^{1}(\Gamma)$ and all the other variables set to zero, we have that $\alpha^{\prime}=\lambda_{2}$ and hence $\alpha \in H^{3 / 2}(\Gamma)$. Moreover, testing (2.30) with $\boldsymbol{\eta} \in H_{0}^{1}\left(\Omega_{i}\right)^{2}$ and $z=0$, we obtain

$$
-\operatorname{div}(\boldsymbol{\sigma}(\boldsymbol{\beta}))=\boldsymbol{\gamma} \quad \text { in } \Omega_{i},
$$

where $\boldsymbol{\sigma}(\boldsymbol{\beta}):=\frac{1}{12}\left\{\frac{E}{1+\nu} \boldsymbol{\epsilon}(\boldsymbol{\beta})+\frac{E}{1-\nu^{2}} \operatorname{div} \boldsymbol{\beta}\right\}$ is the plane-strain stress tensor. Thus, testing (2.10) with $\boldsymbol{\eta}$ $\in H_{0}^{1}(\Omega)^{2}$ and all the other test functions set to zero, integrating by parts in each subdomain $\Omega_{i}$ and using the above equation, we arrive at

$$
\int_{\Gamma} \llbracket \boldsymbol{\sigma}(\boldsymbol{\beta}) \boldsymbol{n} \rrbracket_{\Gamma} \cdot \boldsymbol{\eta}+\int_{\Gamma}\left(\lambda_{3} \eta_{2}+\lambda_{4} \eta_{1}\right)=0
$$


Consequently, since $\left.\boldsymbol{\beta}\right|_{\Omega_{i}} \in H^{2}\left(\Omega_{i}\right), i=1,2$, we obtain that $\lambda_{3}, \lambda_{4} \in H^{1 / 2}(\Gamma)$. Therefore, from (2.28) and the analogous expression for $\theta_{y}^{\mathrm{s}}$, we obtain that $\theta_{x}^{\mathrm{s}}$ and $\theta_{y}^{\mathrm{s}}$ are in $H^{5 / 2}(\Gamma)$. Finally from these regularity results, $(2.27)$ and the additional regularity of $\alpha$, we obtain $w^{\mathrm{s}} \in H^{5 / 2}(\Gamma)$ and conclude the proof.

Remark 2.3. According to Proposition 2.2, the solution of problem (2.10)-(2.11) satisfies additional regularity. However, we have not proved that the corresponding norms are bounded independently of $t$. Indeed, we have used that $\lambda_{2}, \lambda_{3}$ and $\lambda_{4}$ belong to $H^{-1 / 2}(\Gamma)$, which is true but we do not have bounds of $\left\|\lambda_{2}\right\|_{-1 / 2, \Gamma},\left\|\lambda_{3}\right\|_{-1 / 2, \Gamma}$ and $\left\|\lambda_{4}\right\|_{-1 / 2, \Gamma}$ independent of $t$ (cf. Rem. 1.2). In spite of this, the numerical results in Section 5 seem to suggest that such bounds should hold true.

\section{The Discrete PROBlems}

We consider separately the discretization of the stiffened in-plane and bending problems. Let $\left\{\mathcal{T}_{h}\right\}$ be a regular family of triangulations consistent with $\Gamma$, in the sense that, for all meshes, $\Gamma$ is a union of edges of $\mathcal{T}_{h}$. Let $\mathcal{T}_{h}^{\Gamma}$ be the partition induced by $\mathcal{T}_{h}$ in $\Gamma$. We assume that the family $\left\{\mathcal{T}_{h}\right\}$ is such that $\left\{\mathcal{T}_{h}^{\Gamma}\right\}$ is quasi uniform.

\subsection{Stiffened in-plane plate problem}

We define:

$$
\begin{aligned}
X_{h} & :=\left\{z_{h} \in H_{0}^{1}(\Omega):\left.z_{h}\right|_{T} \in \mathcal{P}_{1} \forall T \in \mathcal{T}_{h}\right\}, \\
P_{k h} & :=\left\{v_{h} \in H_{0}^{1}(\Gamma):\left.v_{h}\right|_{\ell} \in \mathcal{P}_{k} \forall \ell \in \mathcal{T}_{h}^{\Gamma}\right\}, \quad k \in \mathbb{N},
\end{aligned}
$$

where $\mathcal{P}_{k}$ denotes the polynomials of degree at most $k$. Let $\boldsymbol{X}_{h}:=X_{h} \times X_{h}$. The discrete analogue to problem $(2.7)-(2.8)$ reads:

Find $\left(\boldsymbol{u}_{h}, u_{h}^{\mathrm{s}}\right) \in \boldsymbol{X}_{h} \times P_{1 h}$ and $\lambda_{1 h} \in P_{1 h}$ such that

$$
\begin{aligned}
a_{1}\left(\boldsymbol{u}_{h}, \boldsymbol{v}_{h}\right)+\int_{\Gamma} \frac{\mathrm{d} u_{h}^{\mathrm{s}}}{\mathrm{d} x} \frac{\mathrm{d} v_{h}^{\mathrm{s}}}{\mathrm{d} x}+\int_{\Gamma} \lambda_{1 h}\left(v_{1 h}-v_{h}^{\mathrm{s}}\right) & =\int_{\Omega} \boldsymbol{f} \cdot \boldsymbol{v}_{h} \quad \forall\left(\boldsymbol{v}_{h}, v_{h}^{\mathrm{s}}\right) \in \boldsymbol{X}_{h} \times P_{1 h}, \\
\int_{\Gamma} \chi_{1 h}\left(u_{1 h}-u_{h}^{\mathrm{s}}\right) & =0 \quad \forall \chi_{1 h} \in P_{1 h} .
\end{aligned}
$$

Since $\boldsymbol{X}_{h} \subset H_{0}^{1}(\Omega)^{2}$ and $P_{1 h} \subset H_{0}^{1}(\Gamma)$, by virtue of (2.9) we only need to prove the following inf-sup condition to be able to apply the classical Babuška-Brezzi theory:

$$
\sup _{0 \neq\left(\boldsymbol{v}_{h}, v_{h}^{\mathrm{s}}\right) \in \boldsymbol{X}_{h} \times P_{1 h}} \frac{\int_{\Gamma} \chi_{h}\left(v_{1 h}-v_{h}^{\mathrm{s}}\right)}{\left\|\boldsymbol{v}_{h}\right\|_{1, \Omega}+\left\|v_{h}^{\mathrm{s}}\right\|_{1, \Gamma}} \geq C\left\|\chi_{h}\right\|_{-1 / 2, \Gamma} \quad \forall \chi_{h} \in P_{1 h} .
$$

To prove this, we will define a Fortin operator $\bar{\Pi}$. With this aim we define several auxiliary operators.

First let $\hat{\pi}: L^{2}(\Gamma) \rightarrow P_{1 h}$ be the $L^{2}$-projection. Clearly we have that

$$
\|\hat{\pi} v\|_{0, \Gamma} \leq C\|v\|_{0, \Gamma} \quad \forall v \in L^{2}(\Gamma) .
$$

Moreover, it is a classical result (see [8], Lem. 1.131) that

$$
\|\hat{\pi} v\|_{1, \Gamma} \leq C\|v\|_{1, \Gamma} \quad \forall v \in H_{0}^{1}(\Gamma),
$$

provided $\left\{\mathcal{T}_{h}^{\Gamma}\right\}$ is a quasi-uniform family of meshes. Consequently, using interpolation of Banach spaces we arrive at

$$
\|\hat{\pi} v\|_{1 / 2, \Gamma} \leq C\|v\|_{1 / 2, \Gamma} .
$$


Next, let $I_{\mathrm{SZ}}: H_{0}^{1}(\Omega) \rightarrow X_{h}$ be a Scott-Zhang interpolation operator (see [17]) such that if $v \in H_{0}^{1}(\Omega)$ and $\left.v\right|_{\Gamma} \in P_{1 h}$, then $\left.\left(I_{\mathrm{Sz}} v\right)\right|_{\Gamma}=\left.v\right|_{\Gamma}$.

On the other hand, let $E: H_{00}^{1 / 2}(\Gamma) \rightarrow H_{0}^{1}(\Omega)$ be a continuous right-inverse of the trace operator on $\Gamma$. Notice that

$$
I_{\mathrm{SZ}}\left(E v_{h}\right)=v_{h} \quad \forall v_{h} \in P_{1 h} .
$$

Now we are in the a position to define the Fortin operator

$$
\bar{\Pi}\left(\boldsymbol{v}, v^{\mathrm{s}}\right):=\left(\left(I_{\mathrm{SZ}}\left(E\left(\hat{\pi}\left(\left.v_{1}\right|_{\Gamma}\right)\right)\right), I_{\mathrm{SZ}} v_{2}\right), \hat{\pi} v^{\mathrm{s}}\right) .
$$

As a consequence of (3.3), (3.4) and the boundedness of the operators $E$ and $I_{\mathrm{SZ}}$, we have

$$
\left\|\bar{\Pi}\left(\boldsymbol{v}, v^{\mathrm{s}}\right)\right\|_{H_{0}^{1}(\Omega)^{2} \times H_{0}^{1}(\Gamma)} \leq C\left\{\|\boldsymbol{v}\|_{1, \Omega}+\left\|v^{\mathrm{s}}\right\|_{1, \Gamma}\right\} \quad \forall\left(\boldsymbol{v}, v^{\mathrm{s}}\right) \in H_{0}^{1}(\Omega)^{2} \times H_{0}^{1}(\Gamma) .
$$

Moreover $\bar{\Pi}$ satisfies the commuting diagram property. In fact, because of (3.5),

$$
\int_{\Gamma}\left(v_{1}-I_{\mathrm{SZ}}\left(E\left(\hat{\pi}\left(\left.v_{1}\right|_{\Gamma}\right)\right)\right)\right) \chi_{h}=0 \quad \forall \chi_{h} \in P_{1 h} .
$$

Therefore $\bar{\Pi}$ satisfies the assumptions of the Fortin lemma [8]. Consequently the Babuška-Brezzi theory (see for instance [5]) allows us to prove the next theorem.

Theorem 3.1. Problem (3.1)-(3.2) has a unique solution $\left(\boldsymbol{u}_{h}, u_{h}^{\mathrm{s}}, \lambda_{1 h}\right) \in \boldsymbol{X}_{h} \times P_{1 h} \times P_{1 h}$ and there exists a positive constant $C$ such that, if $\left(\boldsymbol{u}, u^{\mathrm{s}}\right)$ is the solution to problem $(2.7)-(2.8)$, then

$$
\begin{aligned}
\| \boldsymbol{u}- & \boldsymbol{u}_{h}\left\|_{1, \Omega}+\right\| u^{\mathrm{s}}-u_{h}^{\mathrm{s}}\left\|_{1, \Gamma}+\right\| \lambda_{1}-\lambda_{1 h} \|_{-1 / 2, \Gamma} \\
& \leq C\left\{\inf _{\boldsymbol{v}_{h} \in \boldsymbol{X}_{h}}\left\|\boldsymbol{u}-\boldsymbol{v}_{h}\right\|_{1, \Omega}+\inf _{v_{h}^{\mathrm{s}} \in P_{1 h}}\left\|u^{\mathrm{s}}-v_{h}^{\mathrm{s}}\right\|_{1, \Gamma}+\inf _{\chi_{1 h} \in P_{1 h}}\left\|\lambda_{1}-\chi_{1 h}\right\|_{-1 / 2, \Gamma}\right\} .
\end{aligned}
$$

The error estimate above depends as usual on additional regularity of the solution to the continuous problem (2.7)-(2.8). In what follows we derive a simpler form of this problem. By testing $(2.7)$ with $v^{\mathrm{s}} \in H_{0}^{1}(\Gamma)$ and setting the other variables to zero we have

$$
-\frac{\mathrm{d}^{2} u^{\mathrm{s}}}{\mathrm{d} x^{2}}=\lambda_{1} \in H^{-1 / 2}(\Gamma)
$$

and hence, $u^{\mathrm{s}} \in H^{3 / 2}(\Gamma)$. Moreover, from (2.8), $u_{1}=u^{\mathrm{s}}$ on $\Gamma$. On the other hand, using different test functions in (2.7) it can be shown that

$$
\llbracket \boldsymbol{\sigma}(\boldsymbol{u}) \boldsymbol{n} \rrbracket_{\Gamma}=\left[\begin{array}{c}
\lambda_{1} \\
0
\end{array}\right]
$$

where $\boldsymbol{\sigma}(\boldsymbol{u}):=\frac{E}{1+\nu} \boldsymbol{\epsilon}(\boldsymbol{u})+\frac{E \nu}{(1+\nu)(1-2 \nu)}(\operatorname{div} \boldsymbol{u}) \boldsymbol{I}$ is the plane-stress stress tensor. Therefore, $\boldsymbol{u} \in H_{0}^{1}(\Omega)^{2}$ is the solution to the following problem:

$$
\begin{aligned}
-\operatorname{div}(\boldsymbol{\sigma}(\boldsymbol{u})) & =\boldsymbol{f} \in L^{2}\left(\Omega_{i}\right), \quad i=1,2, \\
u_{1} & =u^{\mathrm{s}} \in H_{00}^{1 / 2}(\Gamma) \cap H^{3 / 2}(\Gamma), \\
\llbracket \frac{\partial u_{2}}{\partial n} \rrbracket & =0 \quad \text { on } \Gamma .
\end{aligned}
$$


This can be seen as the coupling of two elliptic problems, one in each subdomain $\Omega_{i}, i=1,2$, with a Dirichlet boundary condition for the component $u_{1}$ and a transmission condition for $u_{2}$. It seems reasonable to expect additional regularity for the solution to this problem. In such a case, $\lambda_{1}=\frac{E}{2(1+\nu)} \llbracket \frac{\partial u_{1}}{\partial y}+\frac{\partial u_{2}}{\partial x} \rrbracket$ would be more regular, too.

\subsection{The stiffened bending plate problem}

To discretize this problem we consider the DL3 element introduced in [7]. For each $T \in \mathcal{T}_{h}$, let $\mu_{1}, \mu_{2}, \mu_{3}$ be its barycentric coordinates. We denote by $\boldsymbol{\tau}_{\boldsymbol{i}}$ a unit tangent vector to the edge $\mu_{i}=0$ and define

$$
\mathbf{p}_{1}=\mu_{2} \mu_{3} \boldsymbol{\tau}_{\mathbf{1}}, \quad \mathbf{p}_{2}=\mu_{1} \mu_{3} \boldsymbol{\tau}_{\mathbf{2}}, \quad \mathbf{p}_{3}=\mu_{1} \mu_{2} \boldsymbol{\tau}_{\mathbf{3}}
$$

Let

$$
\begin{aligned}
\boldsymbol{Y}_{h} & :=\left\{\boldsymbol{\eta} \in H_{0}^{1}(\Omega)^{2}: \boldsymbol{\eta}_{T} \in \mathcal{P}_{1} \oplus\left\langle\mathbf{p}_{1}, \mathbf{p}_{2}, \mathbf{p}_{3}\right\rangle \quad \forall T \in \mathcal{T}_{h}\right\}, \\
W_{h} & :=\left\{v \in H_{0}^{1}(\Omega):\left.v\right|_{T} \in \mathcal{P}_{1} \quad \forall T \in \mathcal{T}_{h}\right\}, \\
P_{0 h} & :=\left\{\varphi \in L^{2}(\Gamma):\left.\varphi\right|_{\ell} \in \mathcal{P}_{0} \quad \forall \ell \in \mathcal{T}_{h}^{\Gamma}\right\}, \\
\boldsymbol{\Gamma}_{h} & :=\left\{\boldsymbol{\eta}_{h} \in H_{0}(\operatorname{rot}, \Omega):\left.\boldsymbol{\eta}_{h}\right|_{T} \in \mathcal{P}_{0}^{2} \oplus \mathcal{P}_{0}\left(\begin{array}{c}
-x_{2} \\
x_{1}
\end{array}\right) \quad \forall T \in \mathcal{T}_{h}\right\},
\end{aligned}
$$

the latter being the rotated Raviart-Thomas space (see [16]). Let $\Pi$ be the rotated Raviart-Thomas interpolant on this space (see [16] again). This operator is well defined in $H^{1}(\Omega)^{2}$. Moreover for $\boldsymbol{\eta} \in H^{1}(\Omega)^{2} \cap H_{0}(\operatorname{rot}, \Omega)$, $\Pi \boldsymbol{\eta} \in \Gamma_{h}$ and there holds

$$
\|\boldsymbol{\eta}-\Pi \boldsymbol{\eta}\|_{0, \Omega} \leq C h\|\boldsymbol{\eta}\|_{1, \Omega}
$$

Let $\pi: L^{2}(\Gamma) \rightarrow P_{0 h}$ be the orthogonal projection onto $P_{0 h}$. It is well known that

$$
\|v-\pi v\|_{0, \Gamma} \leq C h\|v\|_{1, \Gamma} \quad \forall v \in H^{1}(\Gamma)
$$

Let $\tilde{\pi}: L^{2}(\Gamma) \rightarrow P_{2 h}$ be the orthogonal projection onto $P_{2 h}$.

Finally, let

$$
\boldsymbol{H}_{h}:=\boldsymbol{Y}_{h} \times W_{h} \times P_{1 h} \times P_{1 h} \times P_{2 h} \quad \text { and } \quad \boldsymbol{Q}_{h}:=P_{1 h} \times P_{1 h} \times P_{2 h} .
$$

The discrete problem is defined as follows:

Find $\left(\boldsymbol{\beta}_{h}, w_{h}, w_{h}^{\mathrm{s}}, \theta_{x h}^{\mathrm{s}}, \theta_{y h}^{\mathrm{s}}\right) \in \boldsymbol{H}_{h}$ and $\left(\lambda_{2 h}, \lambda_{3 h}, \lambda_{4 h}\right) \in \boldsymbol{Q}_{h}$ such that

$$
\begin{aligned}
& a_{2}\left(\boldsymbol{\beta}_{h}, \boldsymbol{\eta}_{h}\right)+\frac{1}{t^{2}} \int_{\Omega}\left(\nabla w_{h}-\Pi \boldsymbol{\beta}_{h}\right) \cdot\left(\nabla z_{h}-\Pi \boldsymbol{\eta}_{h}\right)+\int_{\Gamma} \frac{\partial \theta_{x h}^{\mathrm{s}}}{\partial x} \frac{\partial \varphi_{x h}^{\mathrm{s}}}{\partial x}+\int_{\Gamma} \frac{\partial \theta_{y h}^{\mathrm{s}}}{\partial x}, \frac{\partial \varphi_{y h}^{\mathrm{s}}}{\partial x} \\
& \quad+\frac{1}{t^{2}} \int_{\Gamma}\left(\frac{\partial z_{h}^{\mathrm{s}}}{\partial x}+\pi \theta_{y h}^{\mathrm{s}}\right)\left(\frac{\partial z_{h}^{\mathrm{s}}}{\partial x}+\pi \varphi_{y h}^{\mathrm{s}}\right)+\int_{\Gamma} \lambda_{2 h}\left(z_{h}-z_{h}^{\mathrm{s}}\right)+\int_{\Gamma} \lambda_{3 h}\left(\eta_{2 h}-\varphi_{x h}^{\mathrm{s}}\right) \\
& \quad+\int_{\Gamma} \lambda_{4 h}\left(\eta_{1 h}-\varphi_{y h}^{\mathrm{s}}\right)=\int_{\Omega} g z_{h} \quad \forall\left(\boldsymbol{\eta}_{h}, z_{h}, z^{\mathrm{s}}, \varphi_{x h}^{\mathrm{s}}, \varphi_{y h}^{\mathrm{s}}\right) \in \boldsymbol{H}_{h}, \\
& \int_{\Gamma} \chi_{2 h}\left(w_{h}-w_{h}^{\mathrm{s}}\right)+\int_{\Gamma} \chi_{3 h}\left(\beta_{2 h}-\theta_{x h}^{\mathrm{s}}\right)+\int_{\Gamma} \chi_{4 h}\left(\beta_{1 h}+\theta_{y h}^{\mathrm{s}}\right)=0 \quad \forall\left(\chi_{2 h}, \chi_{3 h}, \chi_{4 h}\right) \in \boldsymbol{Q}_{h} .
\end{aligned}
$$


The existence and uniqueness of the solution to (3.10)-(3.11) is obtained using the Babuška-Brezzi theory. The ellipticity condition

$$
\begin{array}{r}
a_{2}\left(\boldsymbol{\eta}_{h}, \boldsymbol{\eta}_{h}\right)+\frac{1}{t^{2}} \int_{\Omega} \mid \nabla z_{h}- \\
\geq\left.\boldsymbol{\eta}_{h}\right|^{2}+\frac{1}{t^{2}} \int_{\Gamma}\left(\frac{\partial z_{h}^{\mathrm{s}}}{\partial x}+\pi \varphi_{y h}^{\mathrm{s}}\right)^{2}+\int_{\Gamma}\left|\frac{\partial \varphi_{x h}^{\mathrm{s}}}{\partial x}\right|^{2}+\int_{\Gamma}\left|\frac{\partial \varphi_{y h}^{\mathrm{s}}}{\partial x}\right|^{2} \\
\geq C\left\{\left\|\boldsymbol{\eta}_{h}\right\|_{1, \Omega}^{2}+\left\|z_{h}\right\|_{1, \Omega}^{2}+\left\|z_{h}^{\mathrm{s}}\right\|_{1, \Gamma}^{2}+\left\|\varphi_{x h}^{\mathrm{s}}\right\|_{1, \Gamma}^{2}+\left\|\varphi_{y h}^{\mathrm{s}}\right\|_{1, \Gamma}^{2}\right\} \\
\forall\left(\boldsymbol{\eta}_{h}, z_{h}, z^{\mathrm{s}}, \varphi_{x h}^{\mathrm{s}}, \varphi_{y h}^{\mathrm{s}}\right) \in \boldsymbol{H}_{h},
\end{array}
$$

is obtained from the ellipticity of the discrete unstiffened plate problem (see for instance [7]), the ellipticity of the discrete beam problem (see for instance [1]) and Poincaré inequality in $H_{0}^{1}(\Gamma)$.

On the other hand, it is simple to show that the discrete inf-sup condition holds true (with a positive constant in principle depending of $h$ ) if and only if

$$
\sup _{0 \neq\left(\boldsymbol{\eta}, z_{h}, z_{h}^{\mathrm{s}}, \varphi_{x h}^{\mathrm{s}}, \varphi_{y h}^{\mathrm{s}}\right) \in \boldsymbol{H}_{h}} \int_{\Gamma} \chi_{2 h}\left(z_{h}-z_{h}^{\mathrm{s}}\right)+\chi_{3 h}\left(\eta_{2 h}-\varphi_{x h}^{\mathrm{s}}\right)+\chi_{4 h}\left(\eta_{1 h}+\varphi_{y h}^{\mathrm{s}}\right)>0 \quad \forall\left(\chi_{2 h}, \chi_{3 h}, \chi_{4 h}\right) \in \boldsymbol{Q}_{h} .
$$

The latter can be proved by taking $\chi_{2 h}=\left.z_{h}\right|_{\Gamma}-z_{h}^{\mathrm{s}}, \chi_{3 h}=\left.\eta_{2 h}\right|_{\Gamma}-\varphi_{x h}^{\mathrm{s}}$ and $\chi_{4 h}=\left.\eta_{1 h}\right|_{\Gamma}+\varphi_{y h}^{\mathrm{s}}$. Thus we arrive at the following lemma.

Lemma 3.2. Problem (3.10)-(3.11) has a unique solution.

\section{ERror estimate}

Let us introduce the discrete shear terms approximating (2.26) and (2.27):

$$
\begin{aligned}
\gamma_{h} & :=\frac{1}{t^{2}}\left(\nabla w_{h}-\Pi \boldsymbol{\beta}_{h}\right), \\
\alpha_{h} & :=\frac{1}{t^{2}}\left(\frac{\partial z_{h}^{\mathrm{s}}}{\partial x}+\pi \theta_{y h}^{\mathrm{s}}\right) .
\end{aligned}
$$

The first step to obtain error estimates is to write an error equation.

With this aim, we test the continuous problem (2.10)-(2.11) and the discrete problem (3.10)-(3.11) with $\left(\boldsymbol{\eta}_{h}, z_{h}, z_{h}^{\mathrm{s}}, \varphi_{x h}^{\mathrm{s}}, \varphi_{y h}^{\mathrm{s}}\right) \in \boldsymbol{H}_{h}$ to obtain

$$
\begin{aligned}
& a_{2}\left(\boldsymbol{\beta}-\boldsymbol{\beta}_{h}, \boldsymbol{\eta}_{h}\right)+\int_{\Omega}\left(\boldsymbol{\gamma}-\gamma_{h}\right) \cdot\left(\nabla z_{h}-\Pi \boldsymbol{\eta}_{h}\right)+\int_{\Gamma}\left(\alpha-\alpha_{h}\right)\left(\frac{\partial z^{\mathrm{s}}}{\partial x}+\pi \varphi_{y}^{\mathrm{s}}\right) \\
& \quad+\int_{\Gamma}\left(\frac{\partial \theta_{y}^{\mathrm{s}}}{\partial x}-\frac{\partial \theta_{y h}^{\mathrm{s}}}{\partial x}\right) \frac{\partial \varphi_{y}^{\mathrm{s}}}{\partial x}+\int_{\Gamma}\left(\frac{\partial \theta_{x}^{\mathrm{s}}}{\partial x}-\frac{\partial \theta_{x h}^{\mathrm{s}}}{\partial x}\right) \frac{\partial \varphi_{x}^{\mathrm{s}}}{\partial x}+\int_{\Gamma}\left(\lambda_{2}-\lambda_{2 h}\right)\left(z-z^{\mathrm{s}}\right) \\
& \quad+\int_{\Gamma}\left(\lambda_{3}-\lambda_{3 h}\right)\left(\eta_{2}-\varphi_{x}^{\mathrm{s}}\right)+\int_{\Gamma}\left(\lambda_{4}-\lambda_{4 h}\right)\left(\eta_{1}-\varphi_{y}^{\mathrm{s}}\right) \\
& \quad=\int_{\Omega} \gamma \cdot\left(\boldsymbol{\eta}_{h}-\Pi \boldsymbol{\eta}_{h}\right)+\int_{\Gamma} \alpha\left(\pi \varphi_{y h}^{\mathrm{s}}-\varphi_{y h}^{\mathrm{s}}\right) \quad \forall\left(\boldsymbol{\eta}_{h}, z_{h}, z_{h}^{\mathrm{s}}, \varphi_{x h}^{\mathrm{s}}, \varphi_{y h}^{\mathrm{s}}\right) \in \boldsymbol{H}_{h}, \\
& \int_{\Gamma} \chi_{2 h}\left(w-w_{h}-\left(w^{\mathrm{s}}-w_{h}^{\mathrm{s}}\right)\right)+\int_{\Gamma} \chi_{3 h}\left(\beta_{2}-\beta_{2 h}-\left(\theta_{x}^{\mathrm{s}}-\theta_{x h}^{\mathrm{s}}\right)\right) \\
& \quad+\int_{\Gamma} \chi_{4 h}\left(\beta_{1}-\beta_{1 h}+\theta_{y}^{\mathrm{s}}-\theta_{y h}^{\mathrm{s}}\right)=0 \quad \forall\left(\chi_{2 h}, \chi_{3 h}, \chi_{4 h}\right) \in \boldsymbol{Q}_{h} .
\end{aligned}
$$

The following lemma well be used to prove the error estimates. 
Lemma 4.1. Given $\hat{\boldsymbol{\beta}} \in \boldsymbol{Y}_{h}, \hat{w} \in W_{h}$, let $\hat{\boldsymbol{\gamma}}:=t^{-2}(\nabla \hat{w}-\Pi \hat{\boldsymbol{\beta}}) \in \boldsymbol{\Gamma}_{h}, \hat{\theta}_{x}^{\mathrm{s}}:=\left.\hat{\beta}_{2}\right|_{\Gamma} \in P_{1 h}, \hat{\theta}_{y}^{\mathrm{s}}:=-\left.\hat{\beta}_{1}\right|_{\Gamma} \in P_{2 h}$, $\hat{w}^{\mathrm{s}}:=\left.\hat{w}\right|_{\Gamma} \in P_{1 h}$ and $\hat{\alpha}:=t^{-2}\left(\frac{d \hat{w}^{\mathrm{s}}}{\mathrm{d} x}+\pi \hat{\theta}_{y}^{\mathrm{s}}\right) \in P_{0 h}$. Then,

$$
\begin{aligned}
& \left\|\hat{\boldsymbol{\beta}}-\boldsymbol{\beta}_{h}\right\|_{1, \Omega}+t\left\|\hat{\boldsymbol{\gamma}}-\gamma_{h}\right\|_{0, \Omega}+t\left\|\hat{\alpha}-\alpha_{h}\right\|_{0, \Gamma}+\left\|\hat{\theta}_{x}^{\mathrm{s}}-\theta_{x h}^{\mathrm{s}}\right\|_{1, \Gamma}+\left\|\hat{\theta}_{y}^{\mathrm{s}}-\theta_{y h}^{\mathrm{s}}\right\|_{1, \Gamma} \\
& \quad \leq C\left\{\|\hat{\boldsymbol{\beta}}-\boldsymbol{\beta}\|_{1, \Omega}+t\|\hat{\boldsymbol{\gamma}}-\boldsymbol{\gamma}\|_{0, \Omega}+t\|\hat{\alpha}-\alpha\|_{0, \Gamma}+\left\|\hat{\theta}_{x}^{\mathrm{s}}-\theta_{x}^{\mathrm{s}}\right\|_{1, \Gamma}+\left\|\hat{\theta}_{y}^{\mathrm{s}}-\theta_{y}^{\mathrm{s}}\right\|_{1, \Gamma}+h\|\boldsymbol{\gamma}\|_{0, \Omega}+h\|\alpha\|_{0, \Gamma}\right\} .
\end{aligned}
$$

Proof. Let $\hat{R}: H^{-1 / 2}(\Gamma) \rightarrow P_{1 h}$ and $\tilde{R}: H^{-1 / 2}(\Gamma) \rightarrow P_{2 h}$ be the projectors respectively defined for any $\lambda \in H^{-1 / 2}(\Gamma)$ by

$$
\int_{\Gamma}(\lambda-\hat{R} \lambda) \chi_{h}=0 \quad \forall \chi_{h} \in P_{1 h} \quad \text { and } \quad \int_{\Gamma}(\lambda-\tilde{R} \lambda) \chi_{h}=0 \quad \forall \chi_{h} \in P_{2 h} .
$$

Using this in the error equation (4.3) we have

$$
\begin{aligned}
a_{2}\left(\hat{\boldsymbol{\beta}}-\boldsymbol{\beta}_{h}, \boldsymbol{\eta}_{h}\right)+\int_{\Omega}\left(\hat{\boldsymbol{\gamma}}-\boldsymbol{\gamma}_{h}\right) \cdot\left(\nabla z_{h}-\Pi \boldsymbol{\eta}_{h}\right)+\int_{\Gamma}\left(\hat{\alpha}-\alpha_{h}\right)\left(\frac{\partial z_{h}^{\mathrm{s}}}{\partial x}+\pi \varphi_{y h}^{\mathrm{s}}\right) \\
+\int_{\Gamma}\left(\frac{\partial \hat{\theta}_{x}^{\mathrm{s}}}{\partial x}-\frac{\partial \theta_{x h}^{\mathrm{s}}}{\partial x}\right) \frac{\partial \varphi_{x h}^{\mathrm{s}}}{\partial x}+\int_{\Gamma}\left(\frac{\partial \hat{\theta}_{y}^{\mathrm{s}}}{\partial x}-\frac{\partial \theta_{y h}^{\mathrm{s}}}{\partial x}\right) \frac{\partial \varphi_{y h}^{\mathrm{s}}}{\partial x} \\
=-\int_{\Gamma}\left(\hat{R} \lambda_{2}-\lambda_{2 h}\right)\left(z_{h}-z_{h}^{\mathrm{s}}\right)-\int_{\Gamma}\left(\hat{R} \lambda_{3}-\lambda_{3 h}\right)\left(\eta_{1 h}-\varphi_{y h}^{\mathrm{s}}\right)-\int_{\Gamma}\left(\tilde{R} \lambda_{4}-\lambda_{4 h}\right)\left(\eta_{2 h}+\varphi_{x h}^{\mathrm{s}}\right) \\
\quad+a_{2}\left(\hat{\boldsymbol{\beta}}-\boldsymbol{\beta}, \boldsymbol{\eta}_{h}\right)+\int_{\Omega}(\hat{\boldsymbol{\gamma}}-\boldsymbol{\gamma}) \cdot\left(\nabla z_{h}-\Pi \boldsymbol{\eta}_{h}\right)+\int_{\Gamma}(\hat{\alpha}-\alpha)\left(\frac{\partial z_{h}^{\mathrm{s}}}{\partial x}-\pi \varphi_{y h}^{\mathrm{s}}\right) \\
\quad+\int_{\Gamma}\left(\frac{\partial \hat{\theta}_{x}^{\mathrm{s}}}{\partial x}-\frac{\mathrm{d} \theta_{x}^{\mathrm{s}}}{\mathrm{d} x}\right) \frac{\partial \varphi_{x h}^{\mathrm{s}}}{\partial x}+\int_{\Gamma}\left(\frac{\partial \hat{\theta}_{y}^{\mathrm{s}}}{\partial x}-\frac{\mathrm{d} \theta_{y}^{\mathrm{s}}}{\mathrm{d} x}\right) \frac{\partial \varphi_{y h}^{\mathrm{s}}}{\partial x} \\
\quad+\int_{\Omega} \boldsymbol{\gamma} \cdot\left(\boldsymbol{\eta}_{h}-\Pi \boldsymbol{\eta}_{h}\right)+\int_{\Gamma} \alpha\left(\pi \varphi_{y h}^{\mathrm{s}}-\varphi_{y h}^{\mathrm{s}}\right) .
\end{aligned}
$$

Now, take $\boldsymbol{\eta}_{h}=\hat{\boldsymbol{\beta}}-\boldsymbol{\beta}_{h}, z_{h}=\hat{w}-w_{h}, z^{\mathrm{s}}=\hat{w}^{\mathrm{s}}-w_{h}^{\mathrm{s}}, \varphi_{x h}^{\mathrm{s}}=\hat{\theta}_{x}^{\mathrm{s}}-\theta_{x h}^{\mathrm{s}}$ and $\varphi_{y h}^{\mathrm{s}}=\hat{\theta}_{y}^{\mathrm{s}}-\theta_{y h}^{\mathrm{s}}$ and use that $\hat{w}^{\mathrm{s}}=\left.\hat{w}\right|_{\Gamma}$, $\hat{\theta}_{x}^{\mathrm{s}}=\left.\hat{\beta}_{1}\right|_{\Gamma}, \hat{\theta}_{y}^{\mathrm{s}}=-\left.\hat{\beta}_{2}\right|_{\Gamma}$ and (4.4), to obtain

$$
\begin{aligned}
& \int_{\Gamma}\left(\hat{R} \lambda_{2}-\lambda_{2 h}\right)\left(\hat{w}-w_{h}-\left(\hat{w}^{\mathrm{s}}-w_{h}^{\mathrm{s}}\right)\right)+\int_{\Gamma}\left(\hat{R} \lambda_{3}-\lambda_{3 h}\right)\left(\hat{\beta}_{1}-\beta_{1 h}-\left(\hat{\theta}_{x}^{\mathrm{s}}-\theta_{x h}^{\mathrm{s}}\right)\right) \\
& \quad+\int_{\Gamma}\left(\tilde{R} \lambda_{4}-\lambda_{4 h}\right)\left(\hat{\beta}_{2}-\beta_{2 h}+\hat{\theta}_{y}^{\mathrm{s}}-\theta_{y h}^{\mathrm{s}}\right)=0 .
\end{aligned}
$$

Hence, using the ellipticity of $a_{2}$ (cf. (1.15)), the definition of $\hat{\gamma}$ and $\hat{\alpha},(4.1),(4.2)$ and a little of algebra, we obtain

$$
\begin{aligned}
\left\|\hat{\boldsymbol{\beta}}-\boldsymbol{\beta}_{h}\right\|_{1, \Omega}^{2}+t^{2}\left\|\hat{\boldsymbol{\gamma}}-\gamma_{\boldsymbol{h}}\right\|_{0, \Omega}^{2}+t^{2}\left\|\hat{\alpha}-\alpha_{h}\right\|_{0, \Gamma}^{2}+\left\|\hat{\theta}_{y}^{\mathrm{s}}-\theta_{y h}^{\mathrm{s}}\right\|_{1, \Gamma}^{2}+\left\|\hat{\theta}_{x}^{\mathrm{s}}-\theta_{x h}^{\mathrm{s}}\right\|_{1, \Gamma}^{2} \\
\leq C\left\{\|\hat{\boldsymbol{\beta}}-\boldsymbol{\beta}\|_{1, \Omega}^{2}+t^{2}\|\hat{\boldsymbol{\gamma}}-\gamma\|_{0, \Omega}^{2}+t^{2}\|\hat{\alpha}-\alpha\|_{0, \Gamma}^{2}+C_{2}\left\|\hat{\theta}_{y}^{\mathrm{s}}-\theta_{y}^{\mathrm{s}}\right\|_{1, \Gamma}^{2}+\left\|\hat{\theta}_{x}^{\mathrm{s}}-\theta_{x}^{\mathrm{s}}\right\|_{1, \Gamma}^{2}\right. \\
\quad+\|\gamma\|_{0, \Omega}\left\|\left(\hat{\boldsymbol{\beta}}-\boldsymbol{\beta}_{h}\right)-\Pi\left(\hat{\boldsymbol{\beta}}-\boldsymbol{\beta}_{h}\right)\right\|_{0, \Omega}+\|\alpha\|_{0, \Gamma}\left\|\left(\hat{\theta}_{y}^{\mathrm{s}}-\theta_{y h}^{\mathrm{s}}\right)-\pi\left(\hat{\theta}_{y}^{\mathrm{s}}-\theta_{y h}^{\mathrm{s}}\right)\right\|_{0, \Gamma} .
\end{aligned}
$$

where we have used (3.8) and (3.9) to obtain the last two terms. Thus we conclude the lemma. 
Next step consists in defining $\hat{\boldsymbol{\beta}}$ and $\hat{w}$ so that appropriate error estimates hold for the right hand side of (4.5). With this aim we will use the Lagrange interpolant $I_{\mathrm{L}}$ of different functions $\beta_{1}, \beta_{2}, w, \theta_{x}^{\mathrm{s}}$, etc. This interpolant is well defined because of the additional regularity proved in Proposition 2.2. Moreover, for each edge $\ell$ of the triangulation, let $\boldsymbol{t}_{\ell}$ be a unit tangent vector and $b_{\ell}$ the piecewise quadratic bubble associated to $\ell$, so that $b_{\ell} \boldsymbol{t}_{\ell}$ is a tangential bubble as defined in (3.7).

To define $\hat{\boldsymbol{\beta}}$ we use the Lagrange interpolant of $\boldsymbol{\beta}$ enriched with quadratic tangential bubbles so that, for all edges $\ell$,

$$
\int_{\ell} \hat{\boldsymbol{\beta}} \cdot \boldsymbol{t}_{\ell}=\int_{\ell} \boldsymbol{\beta} \cdot \boldsymbol{t}_{\ell}
$$

Namely

$$
\hat{\boldsymbol{\beta}}:=I_{\mathrm{L}}(\boldsymbol{\beta})+\sum_{\ell} c_{\ell} \boldsymbol{b}_{\ell} \boldsymbol{t}_{\ell}
$$

with

$$
c_{\ell}:=\frac{6}{|\ell|} \int_{\ell}\left(\boldsymbol{\beta}-I_{\mathrm{L}} \boldsymbol{\beta}\right) \cdot \boldsymbol{t}_{\ell} .
$$

Based on results from [10], it was shown in [7] that

$$
\|\boldsymbol{\beta}-\hat{\boldsymbol{\beta}}\|_{1, \Omega_{i}} \leq C h\|\boldsymbol{\beta}\|_{2, \Omega_{i}}, \quad i=1,2
$$

On the other hand, we use the standard Lagrange interpolant of $w$ to define $\hat{w}$ :

$$
\hat{w}:=I_{\mathrm{L}} w
$$

Our next goal is to estimate $\|\hat{\gamma}-\gamma\|_{0, \Omega}$. Notice that, in spite of the fact that $\gamma \notin H^{1}(\Omega)^{2}$, its rotated RaviartThomas interpolant is well defined because $\left.\gamma\right|_{\Omega_{i}} \in H^{1}\left(\Omega_{i}\right)^{2}$ and the tangential components of $\left.\gamma\right|_{\Omega_{1}}$ and $\left.\gamma\right|_{\Omega_{2}}$ coincide on $\Gamma$ (see [16]). Moreover, an error estimate similar to (3.8) also holds in this case; namely,

$$
\|\gamma-\Pi \gamma\|_{0, \Omega} \leq C h\left(\|\gamma\|_{1, \Omega_{1}}+\|\gamma\|_{1, \Omega_{2}}\right)
$$

On the other hand another well known property of the rotated Raviart-Thomas interpolant is that $\Pi(\nabla w)=$ $\nabla\left(I_{\mathrm{L}} w\right):=\nabla \hat{w}$ (see [16] again). Therefore,

$$
\hat{\gamma}:=\frac{1}{t^{2}}(\nabla \hat{w}-\Pi \boldsymbol{\beta})=\frac{1}{t^{2}} \Pi(\nabla w-\boldsymbol{\beta})=\Pi \boldsymbol{\gamma}
$$

Next, for $\hat{\theta}_{x}^{s}$, since $\theta_{x}^{\mathrm{s}}=\beta_{2}(c f .2 .13)$, we have that

$$
\hat{\theta}_{x}^{\mathrm{s}}:=\left.\hat{\beta}_{2}\right|_{\Gamma}=\left.\left(I_{\mathrm{L}} \beta_{2}\right)\right|_{\Gamma}=I_{\mathrm{L}} \theta_{x}^{\mathrm{s}}
$$

and hence

$$
\left\|\theta_{x}^{\mathrm{s}}-\hat{\theta}_{x}^{\mathrm{s}}\right\|_{1, \Gamma} \leq C h\left\|\theta_{x}^{\mathrm{s}}\right\|_{2, \Gamma}
$$

Analogously, for $\hat{\theta}_{y}^{\mathrm{s}}$ we have

$$
\hat{\theta}_{y}^{\mathrm{s}}:=-\left.\hat{\beta}_{1}\right|_{\Gamma}=-\left.\left(I_{\mathrm{L}} \beta_{1}\right)\right|_{\Gamma}-\sum_{\ell \in \mathcal{T}_{h}^{\Gamma}} c_{\ell} b_{\ell} .
$$


Since $\theta_{y}^{\mathrm{s}}=-\left.\beta_{1}\right|_{\Gamma}(c f .(2.13))$, there holds

$$
\left\|\theta_{y}^{\mathrm{s}}-\hat{\theta}_{y}^{\mathrm{s}}\right\|_{1, \Gamma} \leq\left\|\theta_{y}^{\mathrm{s}}-I_{\mathrm{L}} \theta_{y}^{\mathrm{s}}\right\|_{1, \Gamma}+\left\|\sum_{l \in \mathcal{T}_{h}^{\Gamma}} c_{l} b_{l}\right\|_{1, \Gamma} .
$$

The first term on the right hand side above is bounded by means of the standard estimates for the Lagrange interpolant. For the second one we write

$$
\left\|\sum_{\ell \in \mathcal{T}_{h}^{\Gamma}} c_{\ell} b_{\ell}\right\|_{1, \Gamma}^{2}=\sum_{\ell \in \mathcal{T}_{h}^{\Gamma}}\left|c_{\ell}\right|^{2}\left\|b_{\ell}\right\|_{1, \ell}^{2} .
$$

Straightforward computations show that $\left\|b_{\ell}\right\|_{1, \ell}^{2} \leq \frac{C}{|\ell|}$. On the other hand, for the coefficients $c_{\ell}$ we use that $\boldsymbol{\beta} \cdot \boldsymbol{t}_{\ell}=\beta_{1}=-\theta_{y}^{\mathrm{s}}$ on $\ell$. Consequently, $I_{\mathrm{L}} \boldsymbol{\beta} \cdot \boldsymbol{t}_{\ell}=-I_{\mathrm{L}} \theta_{y}^{\mathrm{s}}$, and we have from (4.8)

$$
\left|c_{\ell}\right|^{2} \leq \frac{36}{|\ell|}\left\|\theta_{y}^{\mathrm{s}}-I_{\mathrm{L}} \theta_{y}^{\mathrm{s}}\right\|_{0, \ell}^{2} \leq C|\ell|^{3}\left\|\theta_{y}^{\mathrm{s}}\right\|_{2, \ell}^{2} .
$$

Altogether, we obtain

$$
\left\|\theta_{y}^{\mathrm{s}}-\hat{\theta}_{y}^{\mathrm{s}}\right\|_{1, \Gamma} \leq C h\left\|\theta_{y}^{\mathrm{s}}\right\|_{2, \Gamma} .
$$

It remains to estimate the term $\|\hat{\alpha}-\alpha\|_{0, \Gamma}$ in (4.5). With this aim we will show that $\hat{\alpha}=\pi \alpha$. First, it is simple to show that

$$
\pi \frac{\mathrm{d} w^{\mathrm{s}}}{\mathrm{d} x}=\frac{\mathrm{d}}{\mathrm{d} x}\left(I_{\mathrm{L}} w^{\mathrm{s}}\right)
$$

Hence, from (2.13), (4.10) and the definition of $\hat{w}^{\mathrm{s}}$ given in Lemma 4.1, we obtain

$$
\pi \frac{\mathrm{d} w^{\mathrm{s}}}{\mathrm{d} x}=\frac{\mathrm{d} \hat{w}^{\mathrm{s}}}{\mathrm{d} x}
$$

Secondly we will show that

$$
\pi \theta_{y}^{\mathrm{s}}=\pi \hat{\theta}_{y}^{\mathrm{s}} .
$$

To prove this it is enough to show that $\int_{\ell} \theta_{y}^{\mathrm{s}}=\int_{\ell} \hat{\theta}_{y}^{\mathrm{s}}$ for all $\ell \in \mathcal{T}_{h}^{\Gamma}$, which follows from (2.13), (4.6) and the definition of $\hat{\theta}_{y}^{\mathrm{s}}$ in Lemma 4.1:

$$
\int_{\ell} \theta_{y}^{\mathrm{s}}=-\int_{\ell} \beta_{1}=-\int_{\ell} \hat{\beta}_{1}=\int_{\ell} \hat{\theta}_{y}^{\mathrm{s}}
$$

Therefore, (4.16) and (4.15) lead to

$$
\hat{\alpha}=\frac{1}{t^{2}}\left(\frac{\mathrm{d} \hat{w}^{\mathrm{s}}}{\mathrm{d} x}+\pi \hat{\theta}_{y}^{\mathrm{s}}\right)=\frac{1}{t^{2}} \pi\left(\frac{\mathrm{d} w^{\mathrm{s}}}{\mathrm{d} x}+\theta_{y}^{\mathrm{s}}\right)=\pi \alpha .
$$

Consequently, from (3.9) we have

$$
\|\hat{\alpha}-\alpha\|_{0, \Gamma} \leq C h\|\alpha\|_{1, \Gamma} .
$$

Now, we are in a position to prove the following theorem. 
Theorem 4.2. Let $\left(\boldsymbol{\beta}, w, w^{\mathrm{s}}, \theta_{x}^{\mathrm{s}}, \theta_{y}^{\mathrm{s}}\right)$ and $\left(\boldsymbol{\beta}_{h}, w_{h}, w_{h}^{\mathrm{s}}, \theta_{x h}^{\mathrm{s}}, \theta_{y h}^{\mathrm{s}}\right)$ be the solutions of problems (2.10)-(2.11) and (3.10)-(3.11), respectively. Let $\gamma, \alpha, \gamma_{h}$ and $\alpha_{h}$ be as defined in (2.26), (2.27), (4.1) and (4.2), respectively. Then

$$
\begin{aligned}
& \left\|\boldsymbol{\beta}-\boldsymbol{\beta}_{h}\right\|_{1, \Omega}+\left\|\theta_{x}^{\mathrm{s}}-\theta_{x h}^{\mathrm{s}}\right\|_{1, \Gamma}+\left\|\theta_{y}^{\mathrm{s}}-\theta_{y h}^{\mathrm{s}}\right\|_{1, \Gamma}+t\left\|\boldsymbol{\gamma}-\boldsymbol{\gamma}_{h}\right\|_{0, \Omega}+t\left\|\alpha-\alpha_{h}\right\|_{0, \Gamma} \\
& \leq C h\left\{\|\boldsymbol{\beta}\|_{2, \Omega_{1}}+\|\boldsymbol{\beta}\|_{2, \Omega_{2}}+t\|\boldsymbol{\gamma}\|_{1, \Omega_{1}}+t\|\boldsymbol{\gamma}\|_{1, \Omega_{2}}+\|\boldsymbol{\gamma}\|_{0, \Omega}+\left\|\theta_{x}^{\mathrm{s}}\right\|_{2, \Gamma}+\left\|\theta_{y}^{\mathrm{s}}\right\|_{2, \Gamma}+t\|\alpha\|_{1, \Gamma}+\|\alpha\|_{0, \Gamma}\right\} .
\end{aligned}
$$

Proof. Let $\hat{\beta}$ and $\hat{w}$ be as defined in (4.7) and (4.6), respectively. Let $\hat{\gamma}, \hat{\theta}_{x}^{\mathrm{s}}, \hat{\theta}_{y}^{\mathrm{s}}$ and $\hat{\alpha}$ as in Lemma 4.1 . Adding and subtracting these terms on the left hand side of (4.18), using triangular inequality, Lemma 4.1 and estimates (4.9), (4.11), (4.12), (4.14) and (4.17), we conclude the proof.

Corollary 4.3. Under the same assumptions as in Theorem 4.2, there holds

$$
\begin{aligned}
& \left\|w-w_{h}\right\|_{1, \Omega}+\left\|w^{\mathrm{s}}-w_{h}^{\mathrm{s}}\right\|_{1, \Gamma} \\
& \leq C h\left\{\|\boldsymbol{\beta}\|_{2, \Omega_{1}}+\|\boldsymbol{\beta}\|_{2, \Omega_{2}}+t\|\boldsymbol{\gamma}\|_{1, \Omega_{1}}+t\|\boldsymbol{\gamma}\|_{1, \Omega_{2}}+\|\boldsymbol{\gamma}\|_{0, \Omega}+\left\|\theta_{x}^{\mathrm{s}}\right\|_{2, \Gamma}+\left\|\theta_{y}^{\mathrm{s}}\right\|_{2, \Gamma}+t\|\alpha\|_{1, \Gamma}+\|\alpha\|_{0, \Gamma}\right\} .
\end{aligned}
$$

Proof. From (2.26) and (4.1) we have

$$
\nabla w-\nabla w_{h}=t^{2}\left(\gamma-\gamma_{h}\right)+\boldsymbol{\beta}-\Pi \boldsymbol{\beta}_{h}
$$

Hence,

$$
\begin{aligned}
\left|w-w_{h}\right|_{1, \Omega} & \leq t^{2}\left\|\boldsymbol{\gamma}-\gamma_{h}\right\|_{0, \Omega}+\|\boldsymbol{\beta}-\Pi \boldsymbol{\beta}\|_{0, \Omega}+\left\|\Pi\left(\boldsymbol{\beta}-\boldsymbol{\beta}_{h}\right)\right\|_{0, \Omega} \\
& \leq t^{2}\left\|\boldsymbol{\gamma}-\gamma_{h}\right\|_{0, \Omega}+\|\boldsymbol{\beta}-\Pi \boldsymbol{\beta}\|_{0, \Omega}+C\left\|\boldsymbol{\beta}-\boldsymbol{\beta}_{h}\right\|_{1, \Omega},
\end{aligned}
$$

the latter because of (3.8). Analogously, from (2.27) and (4.2) we have

$$
\frac{\mathrm{d} w^{\mathrm{s}}}{\mathrm{d} x}-\frac{\mathrm{d} w_{h}^{\mathrm{s}}}{\mathrm{d} x}=t^{2}\left(\alpha-\alpha_{h}\right)+\theta_{y}^{\mathrm{s}}-\pi \theta_{y h}^{\mathrm{s}}
$$

and hence

$$
\begin{aligned}
\left|w^{\mathrm{s}}-w_{h}^{\mathrm{s}}\right|_{1, \Gamma} & \leq t^{2}\left\|\alpha-\alpha_{h}\right\|_{0, \Gamma}+\left\|\theta_{y}^{\mathrm{s}}-\pi \theta_{y}^{\mathrm{s}}\right\|_{0, \Gamma}+\left\|\pi\left(\theta_{y}^{\mathrm{s}}-\theta_{y h}^{\mathrm{s}}\right)\right\|_{0, \Gamma} \\
& \leq t^{2}\left\|\alpha-\alpha_{h}\right\|_{0, \Gamma}+\left\|\theta_{y}^{\mathrm{s}}-\pi \theta_{y}^{\mathrm{s}}\right\|_{0, \Gamma}+\left\|\theta_{y}^{\mathrm{s}}-\theta_{y h}^{\mathrm{s}}\right\|_{0, \Gamma} .
\end{aligned}
$$

Therefore, the corollary follows from these estimates, (3.8), (3.9) and Theorem 4.2.

\section{NUMERICAL EXPERIMENTS}

In this section we report some numerical results obtained with a code which implements the method proposed above. The aim of the numerical experimentation is to assess the performance of the method. The error estimate from Theorem 4.2 involves higher order norms of quantities which are known to be bounded (cf. Prop. 2.2). These bound are independent of the thickness $t$ for uncoupled plates [2] and rods [3]. However analogous thicknessindependent bounds have not been proved for a stiffened plate. Therefore, one of the goals of the reported numerical experimentation is to test whether the method is actually locking-free. To solve problem (3.10)-(3.11), 


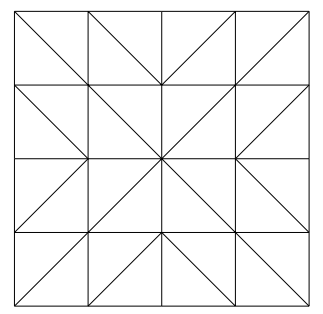

Figure 4. Square plate. Finite element mesh $(N=4)$.

first we eliminate the Lagrange multipliers and impose the constraints by writing the variables corresponding to the stiffener in terms of those of the plate. In fact, (3.11) implies that

$$
w_{h}^{\mathrm{s}}=w_{h}, \quad \theta_{x h}^{\mathrm{s}}=\beta_{2 h}, \quad \theta_{y h}^{\mathrm{s}}=-\beta_{1 h} \quad \text { on } \Gamma .
$$

Thus, problem (3.10)-(3.11) turns out to be equivalent to the following one, in which we included again the physical constants that have been set to 1 for the analysis:

$$
\begin{aligned}
& t^{3} a_{2}\left(\boldsymbol{\beta}_{h}, \boldsymbol{\eta}_{h}\right)+t \int_{\Omega} \kappa \mu\left(\nabla w_{h}-\Pi \boldsymbol{\beta}_{h}\right) \cdot\left(\nabla z_{h}-\Pi \boldsymbol{\eta}_{h}\right)+\int_{\Gamma} E^{\mathrm{s}} I_{y y} \frac{\partial \beta_{1 h}}{\partial x} \frac{\partial \eta_{1 h}}{\partial x} \\
& \quad+\int_{\Gamma} \kappa^{\mathrm{s}} \mu^{\mathrm{s}} A\left(\frac{\partial z_{h}}{\partial x}-\pi \beta_{1 h}\right)\left(\frac{\partial z_{h}}{\partial x}-\pi \eta_{1 h}\right)+\int_{\Gamma} \mu^{\mathrm{s}} J \frac{\partial \beta_{2 h}}{\partial x} \frac{\partial \eta_{2 h}}{\partial x}=t \int_{\Omega} g z_{h}, \quad \forall\left(z_{h}, \boldsymbol{\eta}_{h}\right) \in W_{h} \times \boldsymbol{Y}_{h} .
\end{aligned}
$$

Let us remark that the stiffness matrix of the problem can be easily obtained by static condensation from the separate corresponding stiffness matrices of the plate and the rod. In all tests, we considered a square plate of side length $60 \mathrm{~cm}$. The stiffener crosses the plate joining the mid-points of two opposite edges.

We use uniform meshes obtained by refining the coarse one shown in Figure 4. The parameter $N$ represents the number of elements on each side of the plate. We took $\kappa=\kappa^{\mathrm{s}}=1$ as correction factors in the plate and the stiffener, respectively, for all the tests.

\subsection{Test 1: A free vibration problem for a clamped stiffened plate}

Since no analytical solution for the load problem of the stiffened plate is available to compare with, we used a vibration problem solved in [12] by means of MITC9 elements.

For the vibration problem, we have to consider the mass terms of the plate and the stiffener instead of the load terms. Thus, the vibration problem consists in finding $\omega_{h}>0$ and $\left(w_{h}, \boldsymbol{\beta}_{h}\right) \in W_{h} \times \boldsymbol{Y}_{h}$ such that

$$
\begin{gathered}
t^{3} a_{2}\left(\boldsymbol{\beta}_{h}, \boldsymbol{\eta}_{h}\right)+t \int_{\Omega} \kappa \mu\left(\nabla w_{h}-\Pi \boldsymbol{\beta}_{h}\right) \cdot\left(\nabla z_{h}-\Pi \boldsymbol{\eta}_{h}\right)+\int_{\Gamma} E^{\mathrm{s}} I_{y y} \frac{\partial \beta_{1 h}}{\partial x} \frac{\partial \eta_{1 h}}{\partial x} \\
+\int_{\Gamma} \kappa^{\mathrm{s}} \mu^{\mathrm{s}} A\left(\frac{\partial z_{h}}{\partial x}-\pi \beta_{1 h}\right)\left(\frac{\partial z_{h}}{\partial x}-\pi \eta_{1 h}\right)+\int_{\Gamma} \mu^{\mathrm{s}} J \frac{\partial \beta_{2 h}}{\partial x} \frac{\partial \eta_{2 h}}{\partial x} \\
=\omega_{h}^{2}\left(t \int_{\Omega} \rho w_{h} z_{h}+\frac{t^{3}}{12} \int_{\Omega} \rho \boldsymbol{\beta}_{h} \cdot \boldsymbol{\eta}_{h}+\int_{\Gamma} \rho^{\mathrm{s}} A w_{h} z_{h}+\int_{\Gamma} \rho^{\mathrm{s}} I_{y y} \beta_{1 h} \eta_{1 h}\right. \\
\left.+\int_{\Gamma} \rho^{\mathrm{s}} J \beta_{2 h} \eta_{2 h}\right) \quad \forall\left(z_{h}, \boldsymbol{\eta}_{h}\right) \in W_{h} \times \boldsymbol{Y}_{h},
\end{gathered}
$$

where $\omega_{h}$ is the unknown vibration frequency and $\rho$ and $\rho^{\mathrm{s}}$ are the densities of the plate and the stiffener, respectively. We applied the method to a square clamped stiffened plate with the same physical and geometrical 
TABLE 1. Test 1: Lowest vibration frequencies of a square clamped stiffened plate.

\begin{tabular}{crrrrccc}
\hline Mode & $N=16$ & $N=32$ & $N=48$ & $N=64$ & Order & Extrapolated & {$[12]$} \\
\hline$\omega_{1}$ & 50.919 & 50.527 & 50.452 & 50.426 & 1.97 & 50.392 & 50.36 \\
$\omega_{2}$ & 64.266 & 63.810 & 63.719 & 63.687 & 1.89 & 63.641 & 63.65 \\
$\omega_{3}$ & 76.221 & 75.298 & 75.124 & 75.063 & 1.97 & 74.982 & 74.95 \\
$\omega_{4}$ & 86.656 & 85.694 & 85.510 & 85.445 & 1.95 & 85.358 & 85.36 \\
$\omega_{5}$ & 116.762 & 114.468 & 114.022 & 113.865 & 1.93 & 113.650 & 113.63 \\
$\omega_{6}$ & 123.714 & 121.363 & 120.895 & 120.729 & 1.89 & 120.493 & 120.52 \\
\hline
\end{tabular}

parameters as in [12]:

$$
\begin{aligned}
t & =1 \mathrm{~mm}, \\
E & =E^{\mathrm{s}}=68.85 \times 10^{9} \mathrm{~Pa}, \\
\nu & =\nu^{\mathrm{s}}=0.34, \\
\rho & =\rho^{\mathrm{s}}=2780 \mathrm{~kg} / \mathrm{m}^{3}, \\
A & =67 \mathrm{~mm}^{2}, \\
I_{y y} & =2290 \mathrm{~mm}^{4}, \\
J & =22.33 \mathrm{~mm}^{4} .
\end{aligned}
$$

Table 1 shows the six lowest vibration frequencies computed with the method on four successively refined meshes. The table includes extrapolated frequencies and the order of convergence in powers of $h$ estimated by means of a least squares fitting. It also includes the frequencies computed in [12] with MITC9 elements.

We observe a very good agreement between the values computed with both methods. On the other hand, a quadratic order of convergence can be clearly appreciated. Since typically the order of convergence of the eigenvalues doubles that of the load problem, this corresponds to $O(h)$ for the latter, which is the optimal one for the elements we have used.

\subsection{Test 2: Robustness with respect to the stiffener properties}

The aim of this test is to assess the robustness of the method with respect to the physical parameters of the stiffener.

We considered two asymptotics with respect to the thickness $t$. In the first one, the stiffener rigidity increases as $t$ becomes smaller. As discussed above, the proper scaling for attaining a limit as $t$ goes to zero is to take $E^{\mathrm{s}}$ proportional to $1 / t(c f .(1.12))$. Instead we used $E^{\mathrm{s}}:=E / t^{2}$ for this experiment, so that, in the limit as $t$ goes to zero, this problem corresponds to that of a clamped plate also clamped along its mid-line $\Gamma$.

The material and the stiffener cross-sectional properties were taken as follows:

$$
\begin{aligned}
A & =t^{2}, \\
I_{y y} & =t^{4} / 12, \\
J & =t^{4} / 6, \\
E & =68.85 \times 10^{9} \mathrm{~Pa}, \\
\nu & =\nu^{\mathrm{s}}=0.34 .
\end{aligned}
$$

The load was taken as constant on the whole plate:

$$
g=10^{6} \mathrm{~N} / \mathrm{m}^{4} t^{2} .
$$


TABLE 2. Test 2: Comparison of stiffened plates with an almost rigid and a perfectly rigid stiffener.

\begin{tabular}{clc}
\hline \multirow{2}{*}{ Thickness (m) } & $\frac{\left|w_{h}-w_{h}^{\mathrm{c}}\right|_{1, \Omega}}{\left|w_{h}^{\mathrm{c}}\right|_{1, \Omega}}$ & $\frac{\left|\boldsymbol{\beta}_{h}-\boldsymbol{\beta}_{h}^{\mathrm{c}}\right|_{1, \Omega}}{\left|\boldsymbol{\beta}_{h}^{\mathrm{c}}\right|_{1, \Omega}}$ \\
\hline $10^{-2}$ & $2.6914 \times 10^{-2}$ & $1.6122 \times 10^{-2}$ \\
$10^{-3}$ & $2.8980 \times 10^{-3}$ & $1.6167 \times 10^{-3}$ \\
$10^{-4}$ & $2.9001 \times 10^{-4}$ & $1.6178 \times 10^{-4}$ \\
$10^{-5}$ & $2.9351 \times 10^{-5}$ & $1.6279 \times 10^{-5}$ \\
\hline
\end{tabular}

TABle 3. Test 2: Comparison of a stiffened plate with a very soft stiffener and a plate without a stiffener.

\begin{tabular}{clc}
\hline \multirow{2}{*}{ Thickness $(\mathrm{m})$} & $\frac{\left|w_{h}-w_{h}^{\mathrm{f}}\right|_{1, \Omega}}{\left|w_{h}^{\mathrm{f}}\right|_{1, \Omega}}$ & $\frac{\left|\boldsymbol{\beta}_{h}-\boldsymbol{\beta}_{h}^{\mathrm{f}}\right|_{1, \Omega}}{\left|\boldsymbol{\beta}_{h}^{\mathrm{f}}\right|_{1, \Omega}}$ \\
\hline $10^{-2}$ & $1.4452 \times 10^{-2}$ & $1.5399 \times 10^{-2}$ \\
$10^{-3}$ & $1.4624 \times 10^{-3}$ & $1.5650 \times 10^{-3}$ \\
$10^{-4}$ & $1.4643 \times 10^{-4}$ & $1.5687 \times 10^{-4}$ \\
$10^{-5}$ & $1.5688 \times 10^{-5}$ & $1.6183 \times 10^{-5}$ \\
\hline
\end{tabular}

We took various decreasing values of the thickness ranging from 10 to $0.01 \mathrm{~mm}$. We compared the transverse displacement $w_{h}$ and the rotation $\boldsymbol{\beta}_{h}$ computed with the present method with those corresponding to the plate clamped along $\Gamma$, which were computed by DL3 elements [7]. We denote the latter by $w_{h}^{\mathrm{c}}$ and $\boldsymbol{\beta}_{h}^{\mathrm{c}}$. We measure the deviation between both models by means of the relative differences

$$
\frac{\left|w_{h}-w_{h}^{\mathrm{c}}\right|_{1, \Omega}}{\left|w_{h}^{\mathrm{c}}\right|_{1, \Omega}} \quad \text { and } \quad \frac{\left|\boldsymbol{\beta}_{h}-\boldsymbol{\beta}_{h}^{\mathrm{c}}\right|_{1, \Omega}}{\left|\boldsymbol{\beta}_{h}^{\mathrm{c}}\right|_{1, \Omega}}
$$

computed on the mesh corresponding to $N=64$. It can be clearly seen that the relative differences decreases linearly with the thickness $t$. Moreover, this experiment shows that the stiffened plate behaves as expected in this limit case.

As a second experiment we took $E^{\mathrm{s}}=E$ and the same values as above for $A, I_{y y}, J, E$ and $\nu$. In this case, as $t$ becomes smaller, the effect of the stiffener tends to disappear. In fact, $A=t^{2}$ corresponds to a rod section with width and height proportional to $t$. In the limit as $t$ goes to zero this problem correspond to that of an unstiffened plate. Once more we took values of $t$ ranging from 10 to $0.01 \mathrm{~mm}$ and we compared the transverse displacements and the rotations computed with the present method with those of the unstiffened plate computed by DL3 elements. We denote the latter by $w_{h}^{\mathrm{f}}$ and $\boldsymbol{\beta}_{h}^{\mathrm{f}}$. We report in Table 3 the relative differences between both models computed again on the mesh corresponding to $N=64$.

Once more, it can be clearly observed a linear dependence with respect to $t$. Once more, the behavior of this softly stiffened plates agrees with what is expected.

On the other hand, Tables 2 and 3 show that the method is thoroughly robust with respect to the physical parameters of the stiffener. Moreover, in both cases the results do not deteriorate as $t$ becomes smaller, which suggests that the method is locking-free.

\subsection{Test 3: Testing the locking-free character of the method}

The main goal of this test is to confirm experimentally that the method is locking-free. In this case we took the scaling $E^{\mathrm{s}}:=E / t$, which allows us to achieve a well posed limit problem. We computed a very accurate approximation of the solution with the mesh corresponding to $N=64$ and we took it as an 'exact' solution. We denote by $w^{\mathrm{e}}$ the corresponding transverse displacement. We estimated the error of the numerical solutions computed on coarse meshes $(N=8,12,16)$ by means of $\left|w^{\mathrm{e}}-w_{h}\right|_{1, \Omega}$. 


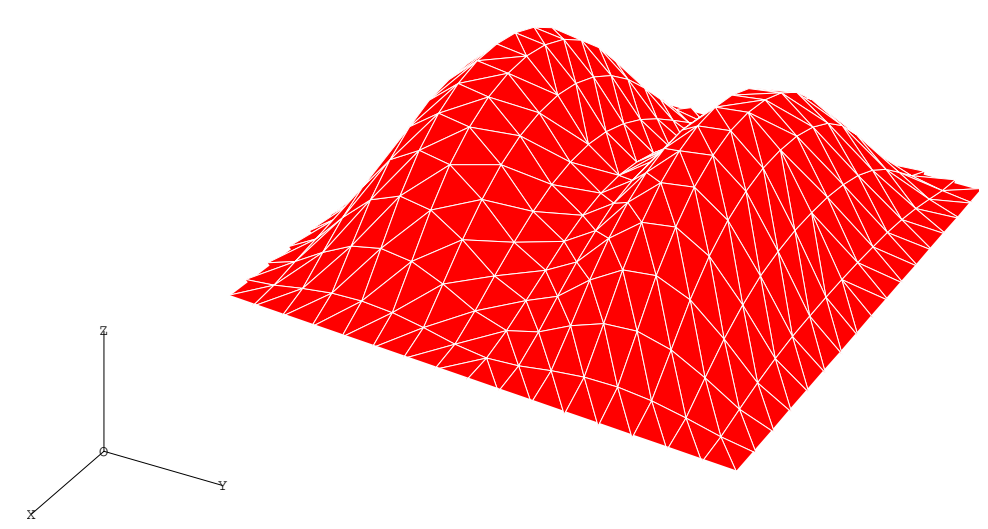

Figure 5. Test 4: Transverse displacement field computed with the proposed method $(N=16)$.

TABLE 4. Test 3: Testing the locking-free character of the method. Computed error $\left|w^{e}-w_{h}\right|_{1, \Omega} \times 10^{8}$ for different plate thickness and meshes.

\begin{tabular}{cccccc}
\hline Thickness $(\mathrm{m})$ & $N=8$ & $N=12$ & $N=16$ & $N=20$ & Order \\
\hline $10^{-2}$ & 0.48539537 & 0.32111497 & 0.26220361 & 0.17661659 & 1.05 \\
$10^{-3}$ & 0.48461630 & 0.32097671 & 0.26194608 & 0.17665166 & 1.05 \\
$10^{-4}$ & 0.48460920 & 0.32097551 & 0.26194343 & 0.17665175 & 1.05 \\
$10^{-5}$ & 0.48460877 & 0.32097521 & 0.26194321 & 0.17665155 & 1.05 \\
\hline$t=0$ (extr.) & 0.48460875 & 0.32097512 & 0.26194319 & 0.17665121 & 1.05 \\
\hline
\end{tabular}

We report in Table 4 the values of $\left|w^{e}-w_{h}\right|_{1, \Omega}$ for different meshes and decreasing values of the thickness. We also include the orders of convergence estimated by means of a least squares fitting and, in the last row, the extrapolated limit values corresponding to $t=0$.

We observe that the method is perfectly locking-free and that an order of convergence close to one is attained even for extremely small values of the thickness. Thus, this experiment provides a solid numerical evidence of the locking-free character of the method.

Figure 5 shows the transverse displacement field of the stiffened plate reported in Table 4 for $t=0.001 \mathrm{~m}$ and $N=16$. In this case the relation $E^{\mathrm{s}} / E=10^{4}$. The effect of the stiffener can be clearly appreciated.

\section{Conclusions}

We analyzed the load problem of the clamped stiffened plate modeled by Reissner-Mindlin equations. We restricted our analysis to the case of concentrically stiffened plates, in which the mid-line of the stiffener lies on the mid-surface of the plate. In such a case, the problem decouples into two well posed problems: a stiffened in-plate plate problem and a stiffened bending plate problem.

We propose a finite element method based on a standard linear triangular elements for the in-plane problem and DL3 elements for the bending problem. The analysis of the former is straightforward, since it turns out a standard elliptic problem not depending on the plate thickness.

The analysis of the bending problem is more involved. We proved optimal order error estimates for all the quantities in terms of higher order norms of these quantities. We proved that these higher order norms are bounded with bounds in principle depending on the plate thickness.

Because of this, the main goal of the numerical experimentation was to assess the locking-free character of the method. The numerical tests also confirmed the optimal order of convergence and showed the robustness of the method with respect to the physical parameters of the stiffener. 
Acknowledgements. RD acknowledges support by ANPCyT under grant PICT 01307 and by Universidad de Buenos Aires under grant X070 (Argentina). RR acknowledges support by FONDAP and BASAL projects CMM, Universidad de Chile (Chile). FS was supported by FONDECYT project 11100261.

\section{REFERENCES}

[1] D.N. Arnold, Discretization by finite element of a model parameter dependent problem. Numer. Math. 37 (1981) 405-421.

[2] D.N. Arnold and R.S. Falk, A uniformly accurate finite element method for the Reissner-Mindlin plate. SIAM J. Numer. Anal. 26 (1989) 1276-1290.

[3] K. Arunakirinathar and B.D. Reddy, Mixed finite element methods for elastic rods of arbitrary geometry. Numer. Math. 64 (1993) 13-43.

[4] K.-J. Bathe, F. Brezzi and S.W. Cho, The MITC7 and MITC9 plate bending elements, Comput. Struct. 32 (1984) $797-814$.

[5] F. Brezzi and M. Fortin, Mixed and Hybrid Finite Element Methods. Springer-Verlag (1991).

[6] F. d'Hennezel, Domain decomposition method and elastic multi-structures: the stiffened plate problem. Numer. Math. 66 (1993) 181-197.

[7] R.G. Durán and E. Liberman, On the mixed finite element methods for the Reissner-Mindlin plate model. Math. Comput. 58 (1992) 561-573.

[8] A. Ern and J.-L. Guermond, Theory and Practice of Finite Elements. Springer-Verlag, New York (2004).

[9] R. Falk, Finite element methods for linear elasticity, in Mixed Finite Elements, Compatibility Conditions, and Applications. Springer-Verlag, Berlin, Heidelberg (2006) 159-194.

[10] V. Girault and P.A. Raviart, Finite Element Methods for Navier-Stokes Equations. Springer-Verlag, Berlin, Heidelberg (1986).

[11] P. Grisvard, Elliptic Problems in Nonsmooth Domains. Pitman (1985).

[12] T.P. Holopainen, Finite element free vibration analysis of eccentrically stiffened plates. Comput. Struct. 56 (1995) $993-1007$.

[13] V. Janowsky, and P. Procházka, The nonconforming finite element method in the problem of clamped plate with ribs. Appl. Math. 21 (1976) 273-289.

[14] A. Mukherjee and M. Mukhopadhyay, Finite element free vibration of eccentrically stiffened plates. Comput. Struct. 30 (1988) $1303-1317$.

[15] J. O'Leary and I. Harari, Finite element analysis of stiffened plates. Comput. Struct. 21 (1985) 973-985.

[16] P.A. Raviart and J.M. Thomas, A mixed finite element method for second order elliptic problems, in Mathematical Aspects of the Finite Element Method. Lecture Notes in Mathematics, Springer, Berlin, Heidelberg (1977) 292-315.

[17] L. Scott and S. Zhang, Finite element interpolation of nonsmooth functions satisfying boundary conditions. Math. Comput. 54 (1990) 483-493. 